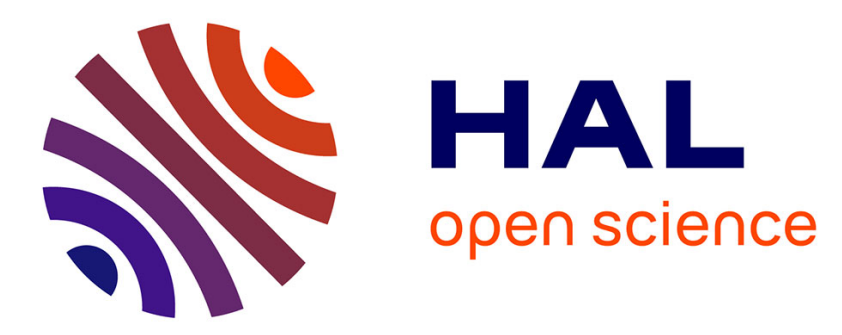

\title{
Synthesis and structure of a heterocyclic ansa pyrrole amino acid
}

\author{
Georg Dirscherl, Parham Rooshenas, Peter R. Schreiner, Frédéric Lamaty,
} Burkhard König

\section{- To cite this version:}

Georg Dirscherl, Parham Rooshenas, Peter R. Schreiner, Frédéric Lamaty, Burkhard König. Synthesis and structure of a heterocyclic ansa pyrrole amino acid. Tetrahedron, 2008, 64 (13), pp.3005-3016. 10.1016/j.tet.2008.01.061 . hal-00258762

\section{HAL Id: hal-00258762 \\ https://hal.science/hal-00258762}

Submitted on 1 Feb 2021

HAL is a multi-disciplinary open access archive for the deposit and dissemination of scientific research documents, whether they are published or not. The documents may come from teaching and research institutions in France or abroad, or from public or private research centers.
L'archive ouverte pluridisciplinaire HAL, est destinée au dépôt et à la diffusion de documents scientifiques de niveau recherche, publiés ou non, émanant des établissements d'enseignement et de recherche français ou étrangers, des laboratoires publics ou privés. 


\title{
Synthesis and structure of a heterocyclic ansa pyrrole amino acid
}

\author{
Georg Dirscherl ${ }^{\mathrm{a}}$, Parham Rooshenas ${ }^{\mathrm{b}}$, Peter R. Schreiner ${ }^{\mathrm{b}}$, \\ Frédéric Lamaty ${ }^{\mathrm{c}}$, Burkhard König ${ }^{\mathrm{a}, *}$ \\ ${ }^{a}$ Institut für Organische Chemie, Universität Regensburg, Universitätsstrasse 31, D-93040 Regensburg, Germany \\ ${ }^{\mathrm{b}}$ Justus-Liebig-University, Institut für Organische Chemie, Heinrich-Buff-Ring 57, D-35392 Giessen, Germany \\ ${ }^{\mathrm{c}}$ Institut des Biomolécules Max Mousseron (IBMM), UMR 5247 CNRS-UM1-UM2, Université de Montpellier 2, \\ Place Eugène Bataillon, 34095 Montpellier Cedex 05, France
}

\begin{abstract}
We report a synthetic route to ansa pyrrole amino acids via olefin ring-closing metathesis of diene precursors in the presence of Grubbs I catalyst. The dienes were prepared by Grignard addition to pyrrole sulfinyl imines. The success of the macrocyclic ring closure depends on the dienes structure and only in the case of the 13-membered compound $\mathbf{2 8}$ sufficient material could be isolated by preparative HPLC separation to investigate its structure spectroscopically. As also rationalized by our computations at the B3LYP/6-311+G(d,p)//B3LYP/6-31G(d) level of theory, $\mathbf{2 8}$ is configurationally stable.
\end{abstract}

\section{Introduction}

Planar chirality arises from stereogenicity resulting from the arrangement of out-of-plane groups with respect to a reference plane. ${ }^{1}$ Structural studies of molecules possessing elements of planar chirality started in the 1940s with the synthesis and resolution of 1,12-dioxa[12]paracyclophane as the first chiral 'ansa' compound. ${ }^{2}$ Lüttringhaus was the first who realized and correctly predicted the existence of enantiomeric ansa compounds. ${ }^{3}$

Compounds displaying planar chirality are found among $\eta^{n}$-olefin metal ${ }^{4}$ and $\eta^{n}$-arene metal complexes, ${ }^{5}$ cyclophanes ${ }^{6}$ and ansa compounds. ${ }^{7}$ Although many of such planar-chiral molecules have been synthesized, only one example of an amino acid with planar chirality exists, at least to the best of our knowledge. Pelter et al. prepared homochiral amino acids, where the chirality depends solely on the chirality of the 4amino-13-carboxy[2.2]paracyclophane. ${ }^{8}$ However, MOPAC computations of simple peptides derived from this amino

\footnotetext{
* Corresponding author.

E-mail address: burkhard.koenig@ @ chemie.uni-regensburg.de (B. König).
}

acid showed that the chain distorts due to the close proximity of the amino and carboxyl groups, so that there was no hydrogen bonding between the second carbonyl and the amide $\mathrm{NH}$ groups. $^{9}$

We report here the synthesis of a planar-chiral amino acid in which the ansa-bridge is formed by an olefin ring-closing metathesis. Scheme 1 shows the general structure of the target heterocyclic amino acids 1 with planar chirality, and the synthetic strategy consisting of ring-closing metathesis (RCM) of suitable dienes $\mathbf{2}$ in the presence of Grubbs' catalyst. The diene precursors were prepared from sulfinyl imines. RCM is a good method for the synthesis of macrocycles ${ }^{10}$ and is also employed as a key step for the synthesis of cyclophane derivatives. ${ }^{11}$ The successful RCM to a 13 -membered ring was reported in the synthesis of the macrotricyclic core of roseophilin $6 .{ }^{12}$ The formation of the rather strained ansachain in this target molecule was supported by conformational control in the precursor bringing the alkene moieties closer together and lowering the enthalpic barrier during ring formation.

Preliminary computations (for a full investigation see below) indicated that such an ansa amino acid would be configurationally stable owing to a rather large barrier for internal 

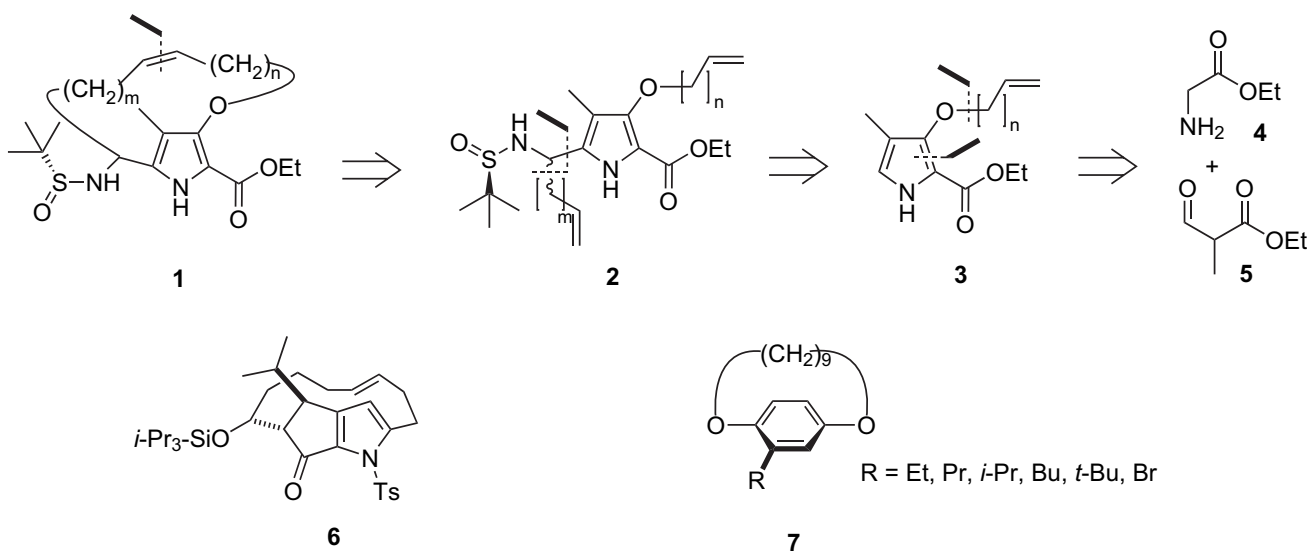

Scheme 1. Top: Retrosynthetic analysis of the target heterocylic amino acids $\mathbf{1}$ with planar chirality. Bottom: Roseophilin $\mathbf{6}$ and planar-chiral cyclophanes $\mathbf{7}$.

rotation. ${ }^{13}$ Studies by König ${ }^{14}$ on the substituent effects on the rotational energy barriers of structurally related planar-chiral cyclophanes 7 using dynamic enantioselective gas chromatography and computer simulation support this reasoning for compound 1: all substituents of compounds 7 were found to be too bulky to permit a rotation of the arene at the experimental conditions $\left(133-145^{\circ} \mathrm{C}\right)$.

\section{Results and discussion}

In earlier studies, we have prepared hydroxypyrrole amino acids (HOPAS $)^{15}$ and incorporated them into small peptides with hairpin structures. ${ }^{16}$ The heterocyclic amino acid is a dipeptide mimic and shows a peptide $\beta$-sheet hydrogen bonding pattern. The chemistry of the HOPAS dipeptide mimic was extended by a palladium catalyzed allylation reaction, which allowed the introduction of a quaternary stereogenic centre. ${ }^{17}$ We now use the dipeptide mimic skeleton in the synthesis of a suitable precursor molecule for RCM.

Ethyl 3-hydroxy-4-methyl-pyrrole-2-carboxylate 8 was prepared as starting material in three steps according to a literature procedure. ${ }^{18}$ The first alkene functionality was introduced by alkylation of the hydroxyl group (Scheme 2). Williamson ether synthesis gave alkenyloxy pyrrole derivatives 9-11 in 61-85\% yield, using potassium carbonate as base and DMF as solvent. Next, pyrrole aldehydes 12-14

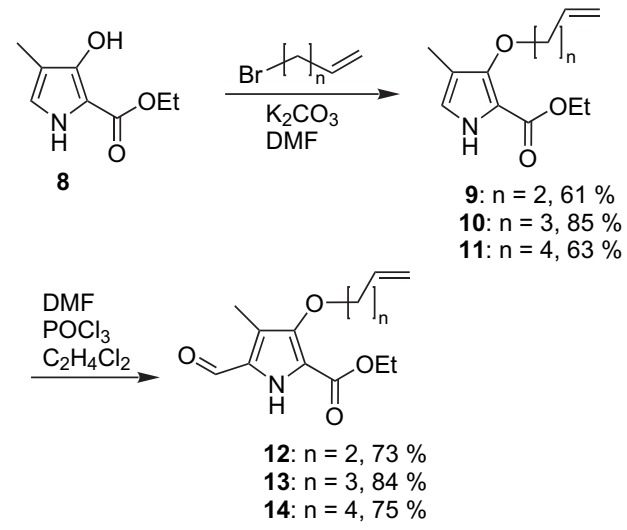

Scheme 2. Synthesis of pyrrole-aldehydes 12-14. were prepared by Vilsmeier-Haack formylation of 9-11 and obtained crystalline in $73-84 \%$ yield.

$\mathrm{N}$-Sulfinyl imines are versatile intermediates in the asymmetric synthesis of chiral amines. Ellman et al. have employed Lewis acidic dehydrating agents $\mathrm{MgSO}_{4}, \mathrm{CuSO}_{4}$ and $\mathrm{Ti}(\mathrm{OEt})_{4}$ for the condensation of $(R)$-tert-butanesulfinamide (15) with aldehydes. ${ }^{19}$ Recently, the formation of sulfinimines by the catalytic action of $\mathrm{Yb}(\mathrm{OTf})_{3}$ was achieved and reaction conditions were extended to Ellman's sulfinyl imines. ${ }^{20}$ We have tested various conditions to convert pyrrole aldehyde $\mathbf{1 3}$ into $(R)$ - $N$-tert-butanesulfinyl aldimines 16-18. $\mathrm{CuSO}_{4}$ was not effective as Lewis acidic dehydrating agent and $\mathrm{Yb}(\mathrm{OTf})_{3}$ did not induce any conversion. With $\mathrm{Ti}(\mathrm{OEt})_{4}$ in dichloromethane the condensation proceeds in excellent yields for all three aldehydes 12-14 (Table 1). The results show the importance to select the right Lewis acid for the conversion of the heterocyclic aldehydes into the corresponding butanesulfinyl aldimines. The structure of compound $\mathbf{1 7}$ was confirmed by an $\mathrm{X}$-ray structure analysis (Fig. 1).

A series of dienes with various chain lengths was prepared via nucleophilic addition of Grignard reagents to imines 1618. The nucleophilic additions of organomagnesium reagents to sulfinyl imines have been explored in detail by Ellman,

Table 1

Synthesis of $N$-tert-butanesulfinyl aldimines 16-18 exploring Lewis acidic dehydrating agents $\mathrm{CuSO}_{4}, \mathrm{Ti}(\mathrm{OEt})_{4}$ and $\mathrm{Yb}(\mathrm{OTf})_{3}$

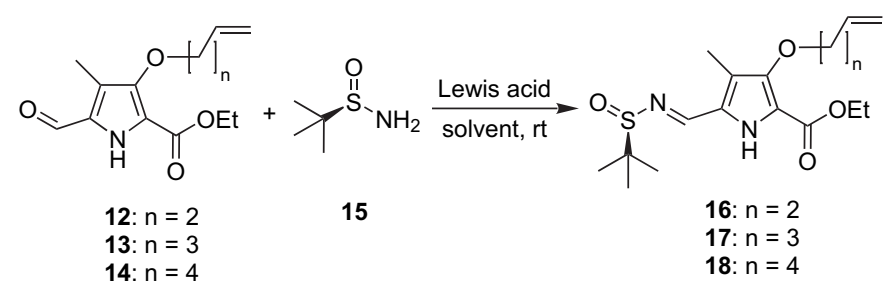

\begin{tabular}{lllllc}
\hline Entry & Aldehyde & Lewis acid & Solvent & Product & Yield (\%) \\
\hline 1 & $\mathbf{1 3}$ & $\mathrm{CuSO}$ & $\mathrm{CH}_{2} \mathrm{Cl}_{2}$ & $\mathbf{1 7}$ & 36 \\
2 & $\mathbf{1 3}$ & $\mathrm{Yb}(\mathrm{OTf})_{3}$ & $\mathrm{THF}$ & $\mathbf{1 7}$ & 0 \\
3 & $\mathbf{1 3}$ & $\mathrm{Ti}(\mathrm{OEt})_{4}$ & $\mathrm{CH}_{2} \mathrm{Cl}_{2}$ & $\mathbf{1 7}$ & 100 \\
4 & $\mathbf{1 2}$ & $\mathrm{Ti}(\mathrm{OEt})_{4}$ & $\mathrm{CH}_{2} \mathrm{Cl}_{2}$ & $\mathbf{1 6}$ & 92 \\
5 & $\mathbf{1 4}$ & $\mathrm{Ti}(\mathrm{OEt})_{4}$ & $\mathrm{CH}_{2} \mathrm{Cl}_{2}$ & $\mathbf{1 8}$ & 91 \\
\hline
\end{tabular}




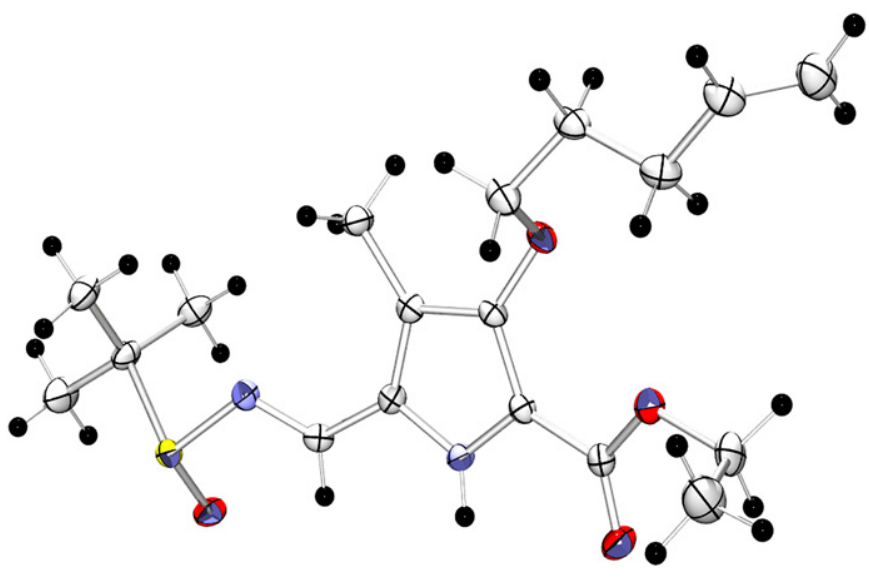

Figure 1. Structure of compound $\mathbf{1 7}$ in the solid state.

Tang and others. ${ }^{21}$ General protocols are described for the addition of alkyl and aryl Grignard reagents to $N$-sulfinyl aldimines with high diastereoselectivity and good yields. Typically, about 2 equiv of Grignard reagents are added at $-78{ }^{\circ} \mathrm{C}$ or $-48{ }^{\circ} \mathrm{C}$ to the $N$-sulfinyl imines to achieve addition, but in our case no nucleophilic addition of the organomagnesium reagents to the sulfinyl imine occurred in various solvents (THF, toluene, diethyl ether, and dichloromethane), even if the reaction temperature was allowed to reach room temperature; the starting material was recovered in all cases. Only if the reaction mixture was heated to $50{ }^{\circ} \mathrm{C}$ nucleophilic addition occurred. The addition products were isolated in moderate to good chemical yields and syn/anti ratios of 9:1 (Table 2).

Table 2

Addition of unsaturated Grignard reagents to $N$-sulfinyl imines 16-18
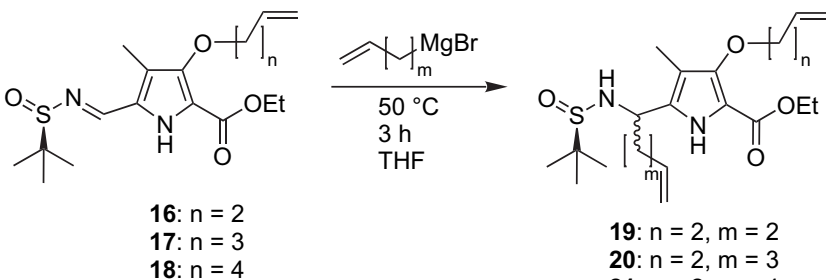

19: $\mathrm{n}=2, \mathrm{~m}=2$

20: $\mathrm{n}=2, \mathrm{~m}=3$

21: $\mathrm{n}=2, \mathrm{~m}=4$

22: $\mathrm{n}=3, \mathrm{~m}=2$

23: $\mathrm{n}=3, \mathrm{~m}=3$

24: $\mathrm{n}=3, \mathrm{~m}=4$

25: $n=4, m=2$

26: $n=4, m=3$

27: $\mathrm{n}=4, \mathrm{~m}=4$

\begin{tabular}{lllll}
\hline Entry & Product & Imine & Yield $^{\mathrm{a}}(\%)$ & $\begin{array}{l}\text { Diastereomeric } \\
\text { ratio }^{\mathrm{b}} \text { (syn:anti) }\end{array}$ \\
\hline 1 & $\mathbf{1 9}$ & $\mathbf{1 6}$ & 61 & $91: 9$ \\
2 & $\mathbf{2 0}$ & $\mathbf{1 6}$ & 63 & $93: 7$ \\
3 & $\mathbf{2 1}$ & $\mathbf{1 6}$ & 64 & $91: 9$ \\
4 & $\mathbf{2 2}$ & $\mathbf{1 7}$ & 68 & $90: 10$ \\
5 & $\mathbf{2 3}$ & $\mathbf{1 7}$ & 71 & $90: 10$ \\
6 & $\mathbf{2 4}$ & $\mathbf{1 7}$ & 65 & $93: 7$ \\
7 & $\mathbf{2 5}$ & $\mathbf{1 8}$ & 68 & $94: 6$ \\
8 & $\mathbf{2 6}$ & $\mathbf{1 8}$ & 50 & $91: 9$ \\
9 & $\mathbf{2 7}$ & $\mathbf{1 8}$ & 72 & $94: 6$ \\
\hline
\end{tabular}

${ }^{a}$ Yields were determined by mass balance of purified material.

${ }^{b}$ Ratios of diastereomers were determined by ${ }^{1} \mathrm{H}$ NMR or HPLC analysis.
Ring-closing diene metathesis has been applied in the synthesis of medium sized rings (9-21-membered ring macrocycles), but yields and optimal conditions (choice of catalyst; solvent) vary and are still difficult to predict. ${ }^{22}$ Therefore, the series of dienes $\mathbf{1 9}-\mathbf{2 7 ^ { 2 3 }}$ with different side chain lengths (Table 2) were reacted with various catalysts. High dilution was used to avoid polymerization via acyclic diene metathesis. Commercially available Grubbs I, Grubbs II and second generation Hoveyda-Grubbs ${ }^{24}$ catalysts were tested in dry and degassed dichloromethane and toluene.

The outcome of the RCM reactions was monitored by TLC, mass spectrometry and HPLC-MS analyses. Only a small fraction of all tested dienes gave macrocyclic products and the best conditions were found using Grubbs I catalyst $(2 \times 15 \mathrm{~mol} \%)$ in high dilution conditions $(0.0005 \mathrm{M})$ in dichloromethane. The formation of the expected macrocyclic structure, although in minor amounts, was indicated by HPLC and mass spectrometry for dienes 23-Boc, 24, 25, 26 and $\mathbf{2 7}$, but only for $\mathbf{2 3}$ the HPLC-MS analysis confirmed amounts of product sufficient for isolation. In the case of diene $\mathbf{2 3}$, besides the 13-membered ansa-compound $\mathbf{2 8}$, a 26-membered macrocycle 29 was obtained as the minor product (Scheme 3). ${ }^{25}$ Both products were isolated by preparative HPLC and investigated in detail by NMR spectroscopy. Isomeric products resulting from the minor anti diastereomer of $\mathbf{2 3}$ were detected analytically, but the amount was too small for isolation. The intramolecular cyclization of compound $\mathbf{2 3}$ to macrocycle 28 (yield: $47 \%$ ) resulted in a 9:1 ratio of Z/E double bond isomers, inseparable by HPLC, favouring the $Z$ configuration. ${ }^{26}$ The observed coupling constant $(J=10.8 \mathrm{~Hz})$ of the olefinic protons in the ${ }^{1} \mathrm{H}$ NMR spectrum confirms this assignment (see Supplementary data). The proton resonance in the open-chain precursor $\mathbf{2 3}$ has a chemical shift of $\delta=5.86$, while in the cyclic form this resonance is shifted downfield by $\Delta \delta=0.8-0.6$. The larger macrocyclic structure 29 (yield: 12\%) was isolated as a mixture of stereoisomers (cis/trans double bonds; head to tail and head to head orientation). Under the reaction conditions and on standing the products loose their tert-butanesulfinamide group.

The stereochemical analysis of the RCM reaction is hindered due to syn/anti-diastereomers of the starting material, diene $\mathbf{2 3}$, and the formation of double bond $E / Z$ isomers in the product. An attempt to reduce the number of isomers by hydrogenation of the double bond was unfortunately not successful.

Density functional theory (DFT) computations at the B3LYP/6-31G(d) level were performed to examine the conformational stability of $\mathbf{2 8}$, more accurate final energies were evaluated using a larger $6-311+\mathrm{G}(\mathrm{d}, \mathrm{p})$ basis set. This approach has shown to provide reasonable results for the conformational energy differences of macrocycles. ${ }^{27}$ The structures K1 and K2 were constructed as simplified models for the two possible atropisomers of $\mathbf{2 8}$. The rotation of the pyrrole ring in the macrocycle $\mathbf{K} \mathbf{1}$ leads to conversion into the other atropisomer K2. This path encompasses transition structure TS1 that is accompanied by an activation enthalpy of $68.1 \mathrm{kcal} / \mathrm{mol}$ at $0 \mathrm{~K}$ (Fig. 2). 

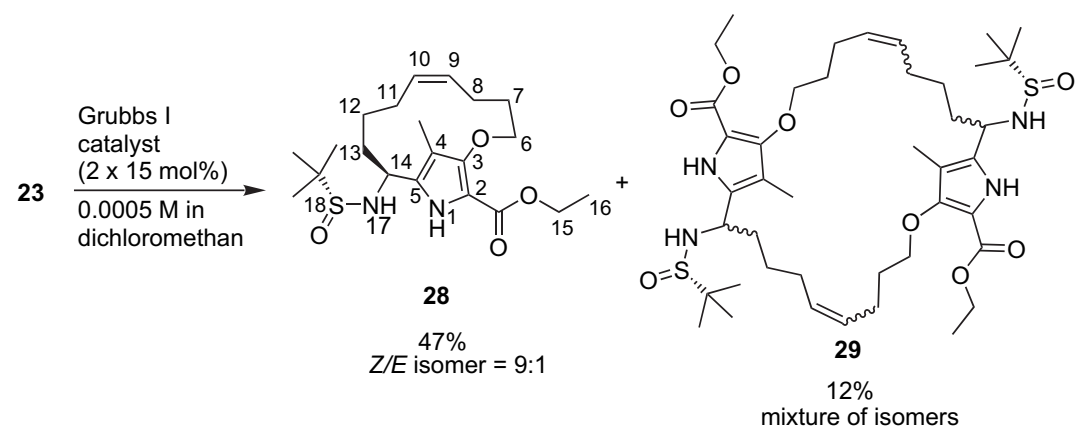

Scheme 3. Synthesis of 13-membered ansa-heterocyclic amino acid $\mathbf{2 8}$ by ring-closing olefin metathesis reaction of compound $\mathbf{2 3}$.

This very high energy barrier emphasizes the conformational stability of $\mathbf{K} \mathbf{1}$ and $\mathbf{K 2}$; it originates entirely from steric effects. During the rotation through the macrocycle, the methyl group demands significant elongation of the $\mathrm{C}-\mathrm{C}$ bonds that nears bond breaking between $\mathrm{C}(33)-\mathrm{C}(30)$ (Fig. 2).

For comparison, we also studied the conversion of the structure $\mathbf{K 3}$, in which the methyl group is replaced by a hydrogen atom. The internal rotation of $\mathbf{K 3}$ to its atropisomer $\mathbf{K} 4$ through transition state TS2 requires an activation enthalpy (via TS2) of merely $6.1 \mathrm{kcal} / \mathrm{mol}$ (Fig. 3). Hence, the K3/K4 couple would not be configurationally stable.

With the reasonable assumption that stable, non-interconverting atropisomers formed, eight product stereoisomers are possible in total. However, the 9:1 ratio of the syn/anti stereoisomers and the $Z / E$ double bond isomers led to significantly different amounts of the product isomers, even in the absence of any stereoinduction in the RCM. Therefore the detection of all minor compounds is difficult or even impossible.

Detailed HPLC-MS analysis of compound $\mathbf{2 8}$ revealed four isomeric compounds: one major isomer, two minor isomers and one isomer in traces (see Supplementary data). The major isomer has $18-R, 14-S$ syn stereochemistry and Zconfiguration of the 9,10-double bond as determined by NMR (see Supplementary data). Using a chiral HPLC, the major isomer peak splits into two peaks in a ratio of about 3:1. This may indicate stereochemical induction of the sulfinylamine and $\mathrm{C}-14$ stereocenters in the ring-closing process. ${ }^{28}$ However, the overall large number of possible isomers and the small amount of product available did not allow the elucidation and assignment of the absolute configuration of the product isomers.

In conclusion, we report a synthetic route to ansa pyrrole amino acids. The ansa-bridge forms by an olefin ring-closing

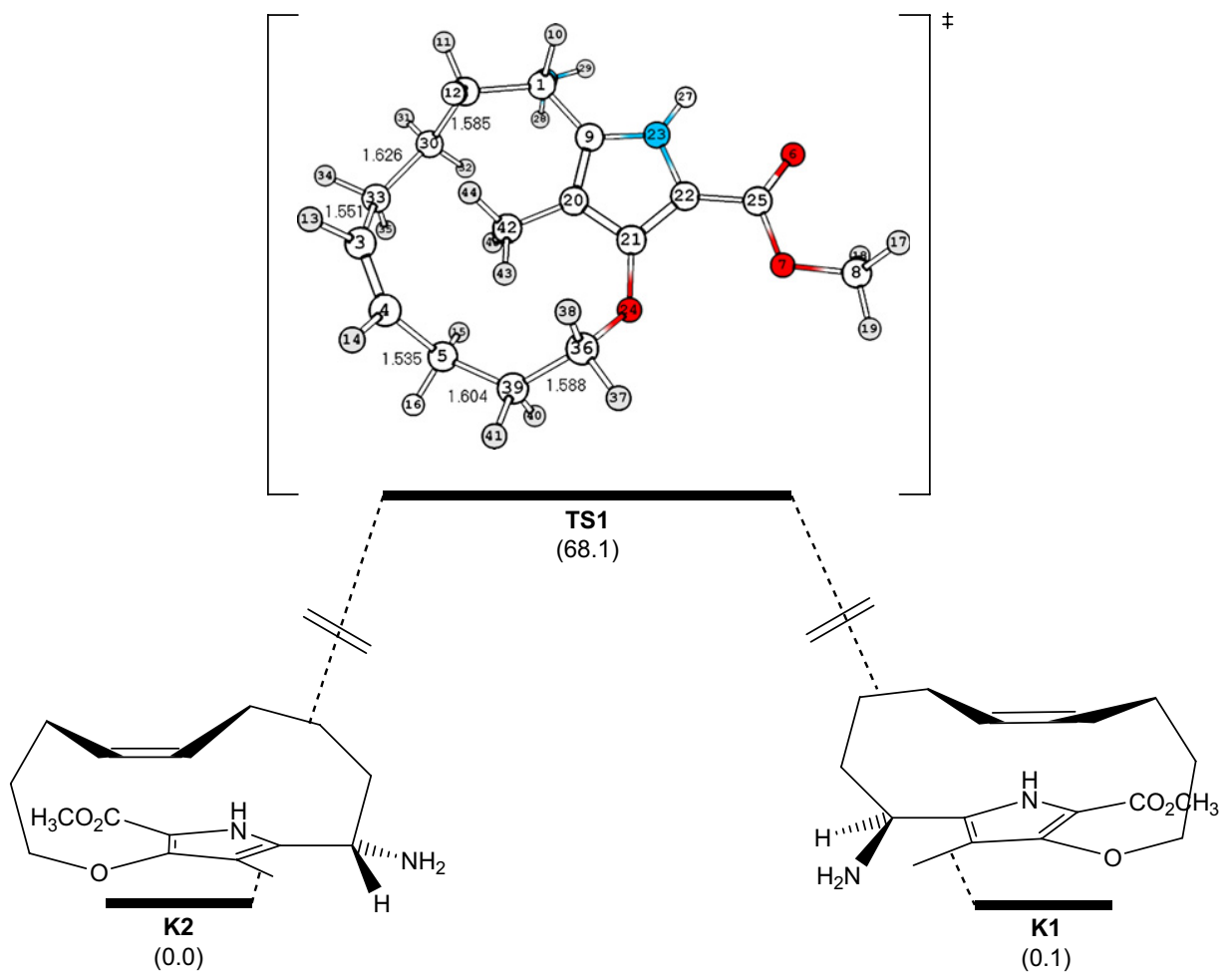

Figure 2. Computed relative enthalpies $\left(\Delta H_{0}\right)$ of structures K1, K2, and TS1 in kcal/mol and optimized structure of TS1 with selected bond lengths in $\AA$ at B3LYP/ 6-311+G(d,p)//B3LYP/6-31G(d) level of the theory. 


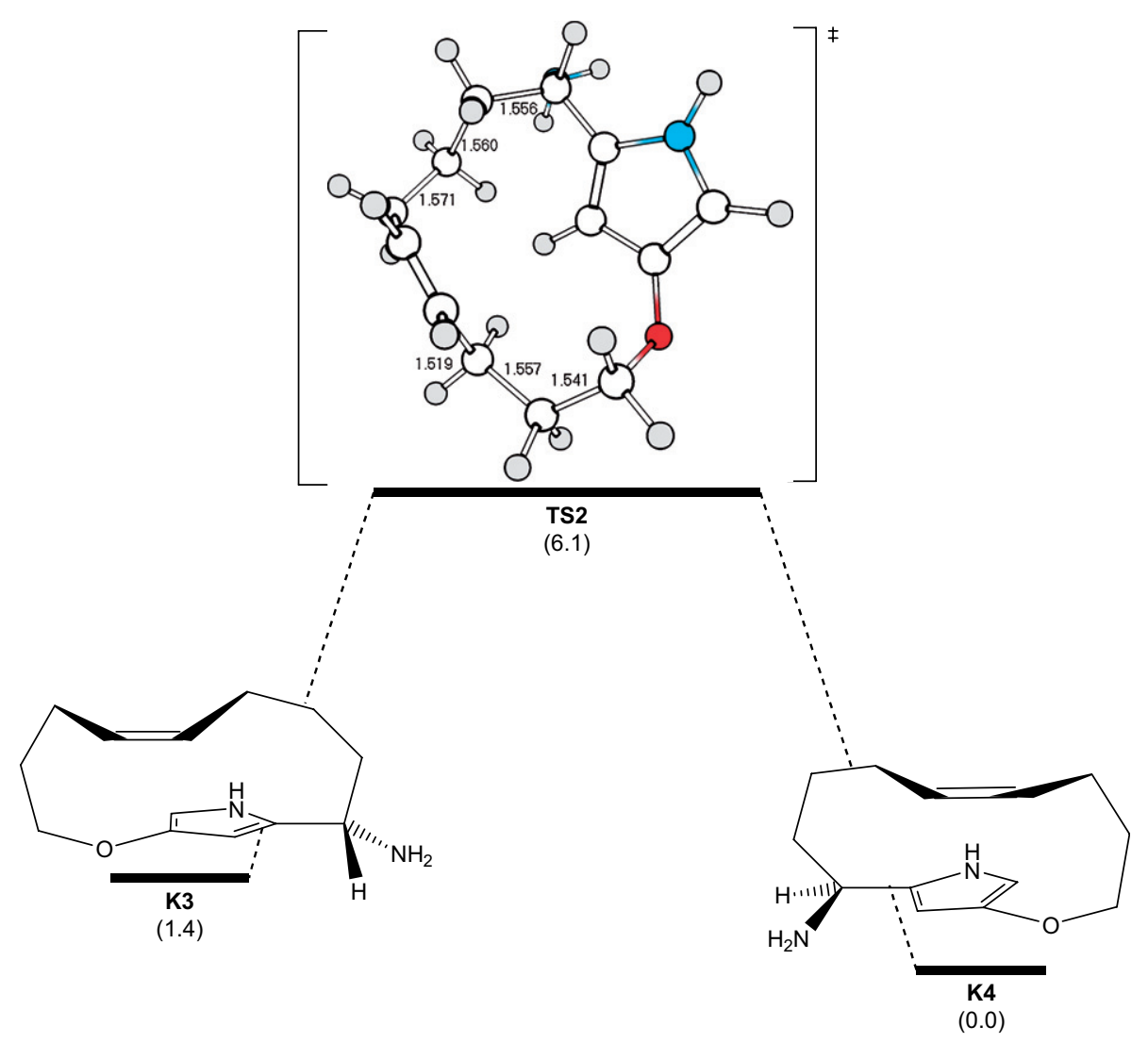

Figure 3. Computed relative enthalpies $\left(\Delta H_{0}\right)$ of structures K3, K4, and TS2 in kcal/mol and optimized structure of TS2 with selected bond lengths in $\AA$ at B3LYP/ 6-311+G(d,p)//B3LYP/6-31G(d) level of the theory.

metathesis reaction of diene precursors in the presence of Grubbs I catalyst. Diene precursors were prepared by Grignard addition to pyrrole sulfinyl imines. Only some of the dienes gave macrocyclic ring-closing products, as identified by HPLC-MS analyses; but yields and selectivities of the ringclosing reactions were low. Only in the case of the 13-membered compound 28 sufficient material could be obtained by preparative HPLC separation to investigate its structure spectroscopically. As also rationalized by our computations at the B3LYP/6-311+G(d,p)//B3LYP/6-31G(d) level of theory, 28 is configurationally stable. Overall, the reported synthetic approach to ansa pyrrole amino acids is feasible, but suffers from poor efficiency of the ring-closing metathesis reaction.

\section{Experimental}

\subsection{Computational methods}

All DFT computations were performed with the Gaussian 03 software package. ${ }^{29}$ Structures were optimized at the DFT level of theory using B3LYP ${ }^{30}$ and a $6-31 \mathrm{G}(\mathrm{d})$ basis set. ${ }^{31}$ Analytical vibrational frequencies were computed at B3LYP/6-31G(d) for all stationary structures to identify minima and transition structures, and to obtain zero-point vibrational energies (ZPVE). The transition structures were confirmed in all cases by the presence of one imaginary frequency. The associated vibration of this frequency was confirmed to correspond to movements in the direction of the reaction coordinate. Additional single-point energies were evaluated at the same level of theory but with a larger basis set $[6-311+\mathrm{G}(\mathrm{d}, \mathrm{p})]$ for all structures utilizing the B3LYP/ 6-31(d) geometries. ${ }^{27}$

\subsubsection{Ethyl 3-(but-3-enyloxy-1H-pyrrole-2-carboxylate) (9)}

To a suspension of $\mathrm{K}_{2} \mathrm{CO}_{3}(364 \mathrm{mg}, 2.63 \mathrm{mmol})$ in dry DMF $(25 \mathrm{~mL})$ was added ethyl 3-hydroxy-4-methyl-pyrrole2-carboxylate $8(420 \mathrm{mg}, 2.48 \mathrm{mmol})$. After the reaction mixture was stirred for $10 \mathrm{~min}$ at room temperature, 4bromo-1-butene $(282 \mu \mathrm{L}, 2.63 \mathrm{mmol})$ was added dropwise and the reaction was stirred for $40 \mathrm{~h}$ at $80{ }^{\circ} \mathrm{C}$. The reaction mixture was quenched with $\mathrm{H}_{2} \mathrm{O}(100 \mathrm{~mL})$ and extracted seven times with each $15 \mathrm{~mL}$ of $\mathrm{CH}_{2} \mathrm{Cl}_{2}$. The collected organic layer ${ }^{27}$ was washed with each $25 \mathrm{~mL}$ of $0.5 \mathrm{M} \mathrm{NaOH}, \mathrm{H}_{2} \mathrm{O}$ and saturated $\mathrm{KHSO}_{4}$ and dried over $\mathrm{MgSO}_{4}$. The solvent was evaporated and the crude product was purified using column chromatography on silica gel $\left(\mathrm{PE} / \mathrm{EtOAc}=8: 2, R_{f}=0.37\right)$ yielding $9(339 \mathrm{mg}, 1.52 \mathrm{mmol}, 61 \%)$ as a colourless oil. ${ }^{1} \mathrm{H}$ NMR $\left(300 \mathrm{MHz}, \mathrm{CDCl}_{3}\right): \delta=1.35$ (t, $\left.{ }^{3} J=7.1,3 \mathrm{H}, \mathrm{H}-12\right)$, $1.96\left(\mathrm{~d},{ }^{4} J=0.8,3 \mathrm{H}, \mathrm{CH}_{3}\right), 2.49\left(\mathrm{ddt},{ }^{3} J=13.6,{ }^{3} J=6.8\right.$, $\left.{ }^{2} J=1.4, \quad 2 \mathrm{H}, \mathrm{H}-7\right), 4.04 \quad\left(\mathrm{t},{ }^{3} J=6.8,2 \mathrm{H}, \mathrm{H}-6\right), 4.29 \quad(\mathrm{q}$, $\left.{ }^{3} J=7.1,2 \mathrm{H}, \mathrm{H}-11\right), 5.00-5.19$ (m, 2H, H-9), 5.91 (ddt, $\left.{ }^{3} J=17.2,{ }^{3} J=10.3,{ }^{3} J=6.8, \quad 1 \mathrm{H}, \quad \mathrm{H}-8\right), 6.54 \quad\left(\mathrm{dd},{ }^{3} J=3.4\right.$, $\left.{ }^{4} J=0.7,1 \mathrm{H}, \mathrm{H}-5\right), 9.03$ (br s, $\left.1 \mathrm{H}, \mathrm{NH}\right) ;{ }^{13} \mathrm{C}$ NMR $(75 \mathrm{MHz}$, $\left.\mathrm{CDCl}_{3}\right): \delta=8.4\left(+, \mathrm{CH}_{3}\right), 14.5(+, \mathrm{C}-12), 34.6(-, \mathrm{C}-7)$, 
60.0 (-, C-11), $74.0(-, \mathrm{C}-6), 111.4\left(\mathrm{C}_{\text {quat }}, \mathrm{C}-2\right), 113.2\left(\mathrm{C}_{\text {quat }}\right.$, C-4), 116.5 (-, C-9), 119.7 (+, C-5), 134.9 (+, C-8), 149.8 $\left(\mathrm{C}_{\text {quat }}, \mathrm{C}-3\right), 160.7\left(\mathrm{C}_{\text {quat }}, \mathrm{C}-10\right)$; IR (neat): $\tilde{\nu}\left[\mathrm{cm}^{-1}\right]=3318$, 3078, 2981, 2933, 2873, 2744, 1668, 1285, 1028; MS $\left(\mathrm{CIMS}, \quad \mathrm{NH}_{3}\right): \mathrm{m} / \mathrm{z}(\%)=224.2 \quad(100) \mathrm{MH}^{+}, 241.2 \quad$ (37) $\left[\mathrm{MNH}_{4}\right]^{+}$; HRMS calcd for $\mathrm{C}_{12} \mathrm{H}_{17} \mathrm{NO}_{3}\left[\mathrm{M}^{+\bullet}\right]$ : 223.1208; found: $223.1206 \pm 0.6 \mathrm{ppm}-\mathrm{C}_{12} \mathrm{H}_{17} \mathrm{NO}_{3}$ (223.27).

\subsubsection{Ethyl 4-methyl-3-(pent-4-enyloxy)-1H-pyrrole- 2-carboxylate (10)}

To a suspension of $\mathrm{K}_{2} \mathrm{CO}_{3}(1.62 \mathrm{~g}, 11.7 \mathrm{mmol})$ in dry DMF $(75 \mathrm{~mL})$ was added ethyl 3-hydroxy-4-methyl-pyrrole-2-carboxylate 8 (1.80 g, $10.6 \mathrm{mmol})$. After the reaction mixture was stirred for $10 \mathrm{~min}$ at room temperature, 5-bromo-1-pentene $(1.39 \mathrm{~mL}, 11.7 \mathrm{mmol})$ was added dropwise and the reaction was stirred for $2 \mathrm{~d}$ at $80^{\circ} \mathrm{C}$. The reaction mixture was quenched with $\mathrm{H}_{2} \mathrm{O}(400 \mathrm{~mL})$ and extracted seven times with each $50 \mathrm{~mL}$ of $\mathrm{CH}_{2} \mathrm{Cl}_{2}$. The collected organic layer was washed with each $100 \mathrm{~mL}$ of $0.5 \mathrm{M} \mathrm{NaOH}, \mathrm{H}_{2} \mathrm{O}$, saturated $\mathrm{KHSO}_{4}$ and dried over $\mathrm{MgSO}_{4}$. The solvent was evaporated and the crude product was purified using column chromatography on silica gel $\left(\mathrm{PE} / \mathrm{EtOAc}=7: 3, R_{f}=0.45\right)$ yielding $10(2.14 \mathrm{~g}, 9.02 \mathrm{mmol}, 85 \%)$ as a colourless oil. ${ }^{1} \mathrm{H}$ NMR $\left(600 \mathrm{MHz}, \mathrm{CDCl}_{3}\right): \delta=1.35\left(\mathrm{t},{ }^{3} J=7.1,3 \mathrm{H}, \mathrm{H}-13\right), 1.86$ (m, 2H, H-7), 1.99 (d, $\left.{ }^{4} J=0.8,3 \mathrm{H}, \mathrm{CH}_{3}\right), 2.28(\mathrm{~m}, 2 \mathrm{H}, \mathrm{H}-8)$, 4.02 (t, $\left.{ }^{3} J=6.5,2 \mathrm{H}, \mathrm{H}-6\right), 4.31$ (q, $\left.{ }^{3} J=7.4,2 \mathrm{H}, \mathrm{H}-12\right), 4.98$ (ddt, ${ }^{3} J=10.2,{ }^{3} J=2.0,{ }^{4} J=1.3,1 \mathrm{H}, \mathrm{H}-10 \mathrm{cis}$ ), $5.05 \quad$ (ddt, $\left.{ }^{3} J=17.2,{ }^{3} J=2.0,{ }^{4} J=1.6,1 \mathrm{H}, \mathrm{H}-10 \operatorname{trans}\right), 5.86$ (ddt, ${ }^{3} J=17.2$, $\left.{ }^{3} J=10.2,{ }^{3} J=6.6,1 \mathrm{H}, \mathrm{H}-9\right), 6.57$ (dq, ${ }^{3} J=3.4,{ }^{4} J=0.8,1 \mathrm{H}, \mathrm{H}-$ 5), $8.58\left(\mathrm{~d},{ }^{3} \mathrm{~J}=3.4,1 \mathrm{H}, \mathrm{NH}\right) ;{ }^{13} \mathrm{C} \mathrm{NMR}\left(150 \mathrm{MHz}, \mathrm{CDCl}_{3}\right)$ : $\delta=8.5\left(+, \mathrm{CH}_{3}\right), 14.5(+, \mathrm{C}-13), 29.4(-, \mathrm{C}-7), 30.2(-, \mathrm{C}-$ 8), 60.0 (-, C-12), 74.2 (-, C-6), 111.5 (C $\left.\mathrm{C}_{\text {quat }}, \mathrm{C}-2\right), 113.3$ (C quat $\left._{1}, \mathrm{C}-4\right), 114.8$ (-, C-10), $119.6(+, \mathrm{C}-5), 138.2(+, \mathrm{C}-$ 9), $149.9\left(\mathrm{C}_{\text {quat }}, \mathrm{C}-3\right), 160.5\left(\mathrm{C}_{\text {quat }}, \mathrm{C}-11\right)$; IR (neat): $\tilde{\nu}$ $\left[\mathrm{cm}^{-1}\right]=3318,3076,2979,2938,2872,2743,1668,1285$, $1029 \mathrm{MS}(\mathrm{EI}, 70 \mathrm{eV}): \mathrm{m} / \mathrm{z}(\%)=237.1(23)\left[\mathrm{M}^{+*}\right], 169.0$ (26) $\left[\mathrm{M}-\mathrm{C}_{4} \mathrm{H}_{8}\right]^{+\bullet}, 123.0(100)\left[\mathrm{M}-\mathrm{C}_{4} \mathrm{H}_{8}-\mathrm{C}_{2} \mathrm{H}_{6} \mathrm{O}\right]^{+\bullet}$; HRMS calcd for $\mathrm{C}_{13} \mathrm{H}_{19} \mathrm{NO}_{3}\left[\mathrm{M}^{+\bullet}\right]$ : 237.1369; found: $237.1363 \pm 2.0 \mathrm{ppm}-$ $\mathrm{C}_{13} \mathrm{H}_{19} \mathrm{NO}_{3}$ (237.30).

\subsubsection{Ethyl 3-(hex-5-enyloxy)-4-methyl-1H-pyrrole- \\ 2-carboxylate (11)}

To a suspension of $\mathrm{K}_{2} \mathrm{CO}_{3}(491 \mathrm{mg}, 3.55 \mathrm{mmol})$ in dry DMF (15 mL) was added ethyl 3-hydroxy-4-methyl-pyrrole2-carboxylate $\mathbf{8}(600 \mathrm{mg}, 3.55 \mathrm{mmol})$. After the reaction mixture was stirred for $10 \mathrm{~min}$ at room temperature, 6bromo-1-hexene $(460 \mu \mathrm{L}, 3.55 \mathrm{mmol})$ was added dropwise and the reaction was stirred for $40 \mathrm{~h}$ at $80{ }^{\circ} \mathrm{C}$. The reaction mixture was quenched with $\mathrm{H}_{2} \mathrm{O}(75 \mathrm{~mL})$ and extracted seven times with each $10 \mathrm{~mL}$ of $\mathrm{CH}_{2} \mathrm{Cl}_{2}$. The collected organic layer was washed with each $20 \mathrm{~mL}$ of $0.5 \mathrm{M} \mathrm{NaOH}, \mathrm{H}_{2} \mathrm{O}$ and saturated $\mathrm{KHSO}_{4}$ and dried over $\mathrm{MgSO}_{4}$. The solvent was evaporated and the crude product was purified using column chromatography on silica gel $\left(\mathrm{PE} / \mathrm{EtOAc}=8: 2, R_{f}=0.40\right)$ yielding $6(565 \mathrm{mg}, 2.25 \mathrm{mmol}, 63 \%)$ as a colourless oil. ${ }^{1} \mathrm{H}$ NMR $\left(300 \mathrm{MHz}, \mathrm{CDCl}_{3}\right): \delta=1.28\left(\mathrm{t},{ }^{3} J=7.1,3 \mathrm{H}, \mathrm{H}-14\right)$, 1.46-1.58 (m, 2H, H-8), 1.64-1.76 (m, 2H, H-7), 1.92 (d, $\left.{ }^{4} J=0.7,3 \mathrm{H}, \mathrm{CH}_{3}\right), 2.00-2.11(\mathrm{~m}, 2 \mathrm{H}, \mathrm{H}-9), 3.94\left(\mathrm{t},{ }^{3} J=6.5\right.$, $2 \mathrm{H}, \mathrm{H}-6), 4.25$ (q, $\left.{ }^{3} J=7.1,2 \mathrm{H}, \mathrm{H}-13\right), 4.83-5.03$ (m, 2H, H11), 5.76 (ddt, ${ }^{3} J=17.0,{ }^{3} J=10.3,{ }^{3} J=6.7,1 \mathrm{H}, \mathrm{H}-10$ ), 6.50 (dd, $\left.{ }^{3} J=3.4,{ }^{4} J=0.7,1 \mathrm{H}, \mathrm{H}-5\right), 8.82$ (br s, $\left.1 \mathrm{H}, \mathrm{NH}\right) ;{ }^{13} \mathrm{C}$ NMR $\left(75 \mathrm{MHz}, \mathrm{CDCl}_{3}\right): \delta=7.5\left(+, \mathrm{CH}_{3}\right), 13.5(+, \mathrm{C}-14), 24.3$ (-, C-8), 28.7 (-, C-7), 32.6 (-, C-9), 58.9 (-, C-13), 73.7 $(-, \mathrm{C}-6), 110.4\left(\mathrm{C}_{\text {quat }}, \mathrm{C}-2\right), 112.2\left(\mathrm{C}_{\text {quat }}, \mathrm{C}-4\right), 113.5(-, \mathrm{C}-$ 11), 118.8 (+, C-5), 137.7 (+, C-10), 149.0 ( $\left.\mathrm{C}_{\text {quat }}, \mathrm{C}-3\right)$, $150.7\left(\mathrm{C}_{\text {quat }}, \mathrm{C}-12\right)$; IR (neat): $\tilde{\nu}\left[\mathrm{cm}^{-1}\right]=3315,2978,2936$, 2868, 1666, 1285, 1028; MS (EI, $70 \mathrm{eV}): \mathrm{m} / \mathrm{z}(\%)=$ 251.3 (11) $\left[\mathrm{M}^{+\bullet}\right], 169.2$ (36) $\left[\mathrm{M}-\mathrm{C}_{6} \mathrm{H}_{10}\right]^{+\bullet}, 123.0$ (100) $\left[\mathrm{M}-\mathrm{C}_{6} \mathrm{H}_{10}-\mathrm{C}_{2} \mathrm{H}_{6} \mathrm{O}\right]^{+\bullet}$; HRMS calcd for $\mathrm{C}_{14} \mathrm{H}_{21} \mathrm{NO}_{3}\left[\mathrm{M}^{+\bullet}\right]$ : 251.1521; found: $251.15 \pm 0.9 \mathrm{ppm}-\mathrm{C}_{14} \mathrm{H}_{21} \mathrm{NO}_{3}$ (251.32).

\subsubsection{Ethyl 3-(but-enyloxy)-5-formyl-4-methyl-1H-pyrrole- \\ 2-carboxylate (12)}

Compound $9(220 \mathrm{mg}, 0.99 \mathrm{mmol})$ in $\mathrm{C}_{2} \mathrm{H}_{4} \mathrm{Cl}_{2}(10 \mathrm{~mL})$ was added dropwise to an ice cooled solution of DMF $(85.5 \mu \mathrm{L}, 1.10 \mathrm{mmol})$ and $\mathrm{POCl}_{3}(101 \mu \mathrm{L}, 1.10 \mathrm{mmol})$ in $\mathrm{C}_{2} \mathrm{H}_{4} \mathrm{Cl}_{2}(10 \mathrm{~mL})$, the mixture was stirred for $30 \mathrm{~min}$, another $30 \mathrm{~min}$ at room temperature and was refluxed for $24 \mathrm{~h} . \mathrm{H}_{2} \mathrm{O}$ $(40 \mathrm{~mL})$ and EtOAc $(20 \mathrm{~mL})$ were added to the cooled mixture and the aqueous layer was extracted three times with each $20 \mathrm{~mL}$ of EtOAc. The combined organic layers were washed three times with $10 \%$ solution of $\mathrm{Na}_{2} \mathrm{CO}_{3}$ and dried over $\mathrm{MgSO}_{4}$. The solvent was evaporated and the crude product was purified using column chromatography on flash-silica gel $\left(\mathrm{PE} / \mathrm{EtOAc}=8: 2, \quad R_{f}=0.21\right) \quad$ yielding $12 \quad(182 \mathrm{mg}$, $0.72 \mathrm{mmol}, 73 \%$ ), as white crystals, $\mathrm{mp}=62{ }^{\circ} \mathrm{C}$; ${ }^{1} \mathrm{H} \mathrm{NMR}$ $\left(300 \mathrm{MHz}, \mathrm{CDCl}_{3}\right): \delta=1.34\left(\mathrm{t},{ }^{3} J=7.1,3 \mathrm{H}, \mathrm{H}-12\right), 2.23(\mathrm{~s}$, $\left.3 \mathrm{H}, \mathrm{CH}_{3}\right), 2.37-2.56(\mathrm{~m}, 2 \mathrm{H}, \mathrm{H}-7), 4.03\left(\mathrm{t},{ }^{3} J=6.7,2 \mathrm{H}, \mathrm{H}-\right.$ 6), $4.32\left(\mathrm{q},{ }^{3} J=7.1,2 \mathrm{H}, \mathrm{H}-11\right), 4.92-5.24$ (m, 2H, H-9), 5.72-6.06 (m, 1H, H-8), 9.20 (br s, 1H, NH), 9.69 (s, 1H, $\mathrm{CHO}) ;{ }^{13} \mathrm{C} \mathrm{NMR}\left(300 \mathrm{MHz}, \mathrm{CDCl}_{3}\right): \delta=6.9\left(+, \mathrm{CH}_{3}\right), 14.3$ (+, C-12), 34.5 (-, C-7), 61.1 (-, C-11), 74.5 (-, C-6), $117.0(-, \mathrm{C}-11), 117.6\left(\mathrm{C}_{\text {quat }}, \mathrm{C}-2\right), 122.6\left(\mathrm{C}_{\text {quat }}, \mathrm{C}-4\right), 127.8$ $\left(\mathrm{C}_{\text {quat }}, \mathrm{C}-5\right), 134.5(+, \mathrm{C}-8), 149.1\left(\mathrm{C}_{\text {quat }}, \mathrm{C}-3\right), 159.6\left(\mathrm{C}_{\text {quat }}\right.$, $\mathrm{C}-10), 179.1$ (+, CHO); IR (KBr): $\tilde{\nu}\left[\mathrm{cm}^{-1}\right]=3442,3261$, 2982, 2928, 2861, 2263, 1676, 1280, 1023; MS (EI, $70 \mathrm{eV})$ : $m / z(\%)=251.2(26)\left[\mathrm{M}^{+\bullet}\right], 151.1(100)\left[\mathrm{M}^{+\cdot}-{ }^{\circ} \mathrm{NH}-(\mathrm{S}=\mathrm{O})\right.$ $\left.\left(\mathrm{CH}_{3}\right)_{3}\right]$; HRMS calcd for $\mathrm{C}_{13} \mathrm{H}_{17} \mathrm{NO}_{4}\left[\mathrm{M}^{+\bullet}\right]$ : 251.1158; found: $251.1162 \pm 1.6 \mathrm{ppm}$.

\subsubsection{Ethyl 5-formyl-4-methyl-3-(pent-4-enyloxy)-1H- pyrrole-2-carboxylate (13)}

Compound 10 (1.94 g, $8.15 \mathrm{mmol})$ in $\mathrm{C}_{2} \mathrm{H}_{4} \mathrm{Cl}_{2}(10 \mathrm{~mL})$ was added dropwise to an ice cooled solution of DMF $(698 \mu \mathrm{L}$, $8.97 \mathrm{mmol})$ and $\mathrm{POCl}_{3}(821 \mu \mathrm{L}, 8.97 \mathrm{mmol})$ in $\mathrm{C}_{2} \mathrm{H}_{4} \mathrm{Cl}_{2}$ $(20 \mathrm{~mL})$, the mixture was stirred for $30 \mathrm{~min}$, another $30 \mathrm{~min}$ at room temperature and was refluxed for $24 \mathrm{~h} . \mathrm{H}_{2} \mathrm{O}$ $(80 \mathrm{~mL})$ and EtOAc $(40 \mathrm{~mL})$ were added to the cooled mixture and the aqueous layer was extracted three times with each $70 \mathrm{~mL}$ EtOAc. The combined organic layers were washed three times with $10 \%$ solution of $\mathrm{Na}_{2} \mathrm{CO}_{3}$ and dried over $\mathrm{MgSO}_{4}$. The solvent was evaporated and the crude product was purified using column chromatography on flash-silica gel (PE/EtOAc $\left.=9: 1, R_{f}=0.40\right)$ yielding $13(1.82 \mathrm{~g}, 6.87 \mathrm{mmol}$, 
$84 \%$ ) as white crystals, $\mathrm{mp}=49{ }^{\circ} \mathrm{C} ;{ }^{1} \mathrm{H} \mathrm{NMR}\left(400 \mathrm{MHz}, \mathrm{CDCl}_{3}\right)$ : $\delta=1.34\left(\mathrm{t},{ }^{3} \mathrm{~J}=7.2,3 \mathrm{H}, \mathrm{H}-13\right), 1.83(\mathrm{~m}, 2 \mathrm{H}, \mathrm{H}-7), 2.22(\mathrm{~m}, 2 \mathrm{H}, \mathrm{H}-$ 8), $2.24\left(\mathrm{~s}, 3 \mathrm{H}, \mathrm{CH}_{3}\right), 3.98\left(\mathrm{t},{ }^{3} J=6.5,2 \mathrm{H}, \mathrm{H}-6\right), 4.34$ (q, ${ }^{3} J=7.2$, $2 \mathrm{H}, \mathrm{H}-12$ ), 4.96 (ddt, ${ }^{3} J=10.2,{ }^{3} J=2.0,{ }^{4} J=1.3,1 \mathrm{H}, \mathrm{H}-10$ cis), 5.02 (ddt, ${ }^{3} J=17.1,{ }^{3} J=2.0,{ }^{4} J=1.6,1 \mathrm{H}, \mathrm{H}-10 \operatorname{trans}$ ), 5.82 (ddt, $\left.{ }^{3} J=17.1,{ }^{3} J=10.2,{ }^{3} J=6.6,1 \mathrm{H}, \mathrm{H}-9\right), 9.55$ (br s, $\left.1 \mathrm{H}, \mathrm{NH}\right), 9.71$ (s, 1H, CHO); ${ }^{13} \mathrm{C} \mathrm{NMR}\left(100 \mathrm{MHz}, \mathrm{CDCl}_{3}\right): \delta=7.0\left(+, \mathrm{CH}_{3}\right)$, 14.3 (+, C-13), 29.3 (-, C-7), 30.1 (-, C-8), 61.1 (-, C-12), 74.7 (-, C-6), 115.0 (+, C-10), $117.7\left(\mathrm{C}_{\text {quat }}, \mathrm{C}-2\right), 122.4\left(\mathrm{C}_{\text {quat }}\right.$, C-4), $128.0\left(\mathrm{C}_{\text {quat }}, \mathrm{C}-5\right), 137.9$ (+, C-9), $149.2\left(\mathrm{C}_{\text {quat }}, \mathrm{C}-3\right)$, $160.5\left(\mathrm{C}_{\text {quat }}, \mathrm{C}-11\right), 179.4(+, \mathrm{CHO}) ; \mathrm{MS}\left(\mathrm{CI}, \mathrm{NH}_{3}\right): \mathrm{m} / \mathrm{z}$ $(\%)=283.3(100)\left[\mathrm{MNH}_{4}\right]^{+}, 266.2(57) \mathrm{MH}^{+}$. Elemental analysis calcd (\%) for $\mathrm{C}_{14} \mathrm{H}_{19} \mathrm{NO}_{4}$ (265.31): C 63.36, H 7.22, N 5.28; found: C 63.16, H 7.57, N 5.21.

\subsubsection{Ethyl 5-formyl-3-(hex-5-enyloxy)-4-methyl-1H- pyrrole-2-carboxylate (14)}

Compound 11 (315 mg, $1.25 \mathrm{mmol})$ in $\mathrm{C}_{2} \mathrm{H}_{4} \mathrm{Cl}_{2}(10 \mathrm{~mL})$ was added dropwise to an ice cooled solution of DMF $(107 \mu \mathrm{L}, 1.38 \mathrm{mmol})$ and $\mathrm{POCl}_{3}(125 \mu \mathrm{L}, 8.97 \mathrm{mmol})$ in $\mathrm{C}_{2} \mathrm{H}_{4} \mathrm{Cl}_{2}(10 \mathrm{~mL})$, the mixture was stirred for $30 \mathrm{~min}$, another $30 \mathrm{~min}$ at room temperature and was refluxed for $24 \mathrm{~h} . \mathrm{H}_{2} \mathrm{O}$ $(40 \mathrm{~mL})$ and EtOAc $(20 \mathrm{~mL})$ were added to the cooled mixture and the aqueous layer was extracted three times with $20 \mathrm{~mL}$ of EtOAc. The combined organic layers were washed three times with $10 \%$ solution of $\mathrm{Na}_{2} \mathrm{CO}_{3}$ and dried over $\mathrm{MgSO}_{4}$. The solvent was evaporated and the crude product was purified using column chromatography on flash-silica gel $(\mathrm{PE} / \mathrm{EtOAc}=9: 1$, $\left.R_{f}=0.41\right)$ yielding $9(265 \mathrm{mg}, 0.95 \mathrm{mmol}, 75 \%)$ as white crystals, $\mathrm{mp}=48{ }^{\circ} \mathrm{C} ;{ }^{1} \mathrm{H} \mathrm{NMR}\left(300 \mathrm{MHz}, \mathrm{CDCl}_{3}\right): \delta=1.38(\mathrm{t}$, $\left.{ }^{3} J=7.1,3 \mathrm{H}, \mathrm{H}-14\right), 1.48-1.87(\mathrm{~m}, 4 \mathrm{H}, \mathrm{H}-7$ and $\mathrm{H}-8), 2.05-$ $2.20(\mathrm{~m}, 2 \mathrm{H}, \mathrm{H}-9), 2.27\left(\mathrm{~s}, 3 \mathrm{H}, \mathrm{CH}_{3}\right), 4.01\left(\mathrm{t},{ }^{3} \mathrm{~J}=6.6,2 \mathrm{H}\right.$, H-6), 4.36 (q, $\left.{ }^{3} J=7.1,2 \mathrm{H}, \mathrm{H}-13\right), 4.90-5.10$ (m, 2H, H-11), 5.72-5.93 (m, 1H, H-10), 9.24 (br s, 1H, NH), 9.73 (s, 1H, $\mathrm{CHO}) ;{ }^{13} \mathrm{C} \mathrm{NMR}\left(75 \mathrm{MHz}, \mathrm{CDCl}_{3}\right): \delta=6.9\left(+, \mathrm{CH}_{3}\right), 14.4$ (+, C-14), 25.3 (-, C-8), 29.5 (-, C-7), 33.5 (-, C-9), 61.1 (-, C-13), 75.3 (-, C-6), 114.7 (-, C-11), $117.6\left(\mathrm{C}_{\text {quat }}, \mathrm{C}-\right.$ 2), $122.5\left(\mathrm{C}_{\text {quat }}, \mathrm{C}-4\right), 127.8\left(\mathrm{C}_{\text {quat }}, \mathrm{C}-5\right), 138.5$ (+, C-10), 149.3 ( $\left.\mathrm{C}_{\text {quat }}, \mathrm{C}-3\right), 159.7$ ( $\left.\mathrm{C}_{\text {quat }}, \mathrm{C}-12\right), 179.1$ (+, CHO); IR $(\mathrm{KBr}): \tilde{\nu}\left[\mathrm{cm}^{-1}\right]=3447,3268,2979,2940,2867,2362,1672$, 1277, 1027; MS (EI, $70 \mathrm{eV}): m / z(\%)=279.3(5)\left[\mathrm{M}^{+\bullet}\right]$, 197.1 (48) $\left[\mathrm{M}-\mathrm{C}_{6} \mathrm{H}_{10}\right]^{+\bullet}, 151.1(100)\left[\mathrm{M}-\mathrm{C}_{6} \mathrm{H}_{10}-\mathrm{C}_{2} \mathrm{H}_{6} \mathrm{O}\right]^{+\bullet}$; HRMS calcd for $\mathrm{C}_{15} \mathrm{H}_{21} \mathrm{NO}_{4}\left[\mathrm{M}^{+\bullet}\right]$ : 279.1471; found: $279.1475 \pm 1.9 \mathrm{ppm}-\mathrm{C}_{15} \mathrm{H}_{21} \mathrm{NO}_{4}$ (279.34).

\subsection{General procedure (GPl) for the synthesis of tert-butanesulfinyl imines}

To a solution of pyrrole aldehyde (1.0 equiv) and $\mathrm{Ti}(\mathrm{OEt})_{4}$ (2.0 equiv) in dry dichloromethane $(5 \mathrm{~mL})$ was added $(R)$-tertbutanesulfinamide (1.2 equiv) under dinitrogen, and the mixture was stirred at the given temperature and time. The reaction mixture was quenched with a mixture of saturated $\mathrm{NH}_{4} \mathrm{Cl}$ solution $(10 \mathrm{~mL})$ and brine $(10 \mathrm{~mL})$ while vigorously stirred. The resulting suspension was filtered through a plug of Celite and the filter cake was washed well with EtOAc $(30 \mathrm{~mL})$. The filtrate was transferred to a separatory funnel, where the organic layer was washed three times with each $10 \mathrm{~mL}$ of brine. The brine layer was extracted once with a small volume of EtOAc and the combined organic portions were dried over $\mathrm{MgSO}_{4}$, filtered and concentrated under vacuum. The sulfinyl imines were purified by silica gel chromatography if no other method is given.

\subsection{1. (R)-Ethyl 3-(but-3-enyloxy)-4-methyl-5-((2-methyl- propan-2-ylsulfinamido)methyl)-1H-pyrrole-2-carboxylate (16)}

Compound 12 (251 mg, $0.64 \mathrm{mmol})$, (R)-tert-butanesulfinamide $(\mathbf{1 5}, 93 \mathrm{mg}, \quad 0.77 \mathrm{mmol})$ and $\mathrm{Ti}(\mathrm{OEt})_{4} \quad(292 \mathrm{mg}$, $1.28 \mathrm{mmol})$ in dry dichloromethane $(5 \mathrm{~mL})$ were allowed to react according to the GP1 at room temperature for $36 \mathrm{~h}$ yielding $152 \mathrm{mg}$ ( $0.43 \mathrm{mmol}$, 92\%, conversion corrected yield, $43 \mathrm{mg}$ of starting material regained $)$ of $\mathbf{1 6}\left(\mathrm{Et}_{2} \mathrm{O} /\right.$ hexanes $=1: 1$; $\left.R_{f}=0.22\right)$ as colourless crystals, $\mathrm{mp}=81{ }^{\circ} \mathrm{C} ;{ }^{1} \mathrm{H} \quad \mathrm{NMR}$ $\left(300 \mathrm{MHz}, \mathrm{CDCl}_{3}\right): \delta=1.25(\mathrm{~s}, 9 \mathrm{H}, t-\mathrm{Bu}), 1.40\left(\mathrm{t},{ }^{3} J=7.1\right.$, $3 \mathrm{H}, \mathrm{H}-12), 2.21$ (s, 3H, $\mathrm{CH}_{3}$ ), 2.52-2.55 (m, 2H, H-7), 4.06 $\left(\mathrm{t},{ }^{3} J=6.8,2 \mathrm{H}, \mathrm{H}-6\right), 4.39$ (q, $\left.{ }^{3} J=7.1, \mathrm{H}-11\right), 5.09-5.21(\mathrm{~m}$, 2H, H-9), 5.92-5.94 (m, 1H, H-8), 8.47 (s, 1H, CHN), 9.28 (br s, $1 \mathrm{H}, \mathrm{NH}) ;{ }^{13} \mathrm{C} \mathrm{NMR}\left(75 \mathrm{MHz}, \mathrm{CDCl}_{3}\right): \delta=7.5\left(+, \mathrm{CH}_{3}\right)$, 14.4 (+, C-12), $22.5(+, t$-Bu), 34.5 (-, C-7), 57.8 (+, $t$-Bu), 60.9 (-, C-11), 74.4 (-, C-6), 116.1 (C $\left.\mathrm{C}_{\text {quat }}, \mathrm{C}-2\right), 116.9$ (-, C9), $120.2\left(\mathrm{C}_{\text {quat }}, \mathrm{C}-4\right), 126.2\left(\mathrm{C}_{\text {quat }}, \mathrm{C}-5\right), 134.5(+, \mathrm{C}-8), 149.5$ $\left(\mathrm{C}_{\text {quat }}, \mathrm{C}-3\right), 150.5(+, \mathrm{CHN}), 160.1\left(\mathrm{C}_{\text {quat }}, \mathrm{C}-10\right)$; IR $(\mathrm{KBr}): \tilde{\nu}$ $\left[\mathrm{cm}^{-1}\right]=3447,3256,2980,2959,2926,2868,1701,1593$, 1272, 1059, 744; MS (FAB+): $m / z(\%)=355(100)[\mathrm{MH}]^{+}$, 289 (43) $\left[\mathrm{M}-\mathrm{C}_{4} \mathrm{H}_{8}\right]^{+}$; HRMS calcd for $\mathrm{C}_{17} \mathrm{H}_{27} \mathrm{~N}_{2} \mathrm{O}_{4} \mathrm{~S}^{+}$: 355.1697; found: $355.1692+1.5 \mathrm{ppm}-\mathrm{C}_{17} \mathrm{H}_{26} \mathrm{~N}_{2} \mathrm{O}_{4} \mathrm{~S}$ (354.46).

\subsection{2. (R)-Ethyl 4-methyl-5-[(2-methylpropan-2-ylsulfin- amido)-methyl]-3-pent-4-enyloxy-1H-pyrrole-2- carboxylate (17)}

Compound 13 (450 mg, $1.70 \mathrm{mmol})$, (R)-tert-butanesulfinamide $(\mathbf{1 5}, 247 \mathrm{mg}, 2.04 \mathrm{mmol})$ and $\mathrm{Ti}(\mathrm{OEt})_{4} \quad(776 \mathrm{mg}$, $3.4 \mathrm{mmol}$ ) in dry dichloromethane were allowed to react according to the GP1 at $35{ }^{\circ} \mathrm{C}$ for $48 \mathrm{~h}$ yielding $625 \mathrm{mg}$ (quantitative) of $17\left(\mathrm{Et}_{2} \mathrm{O} /\right.$ hexanes $\left.=1: 1 ; R_{f}=0.32\right)$ as colourless crystals, $\mathrm{mp}=79^{\circ} \mathrm{C} ;{ }^{1} \mathrm{H} \mathrm{NMR}\left(400 \mathrm{MHz}, \mathrm{CDCl}_{3}\right): \delta=1.24$ $(\mathrm{s}, 9 \mathrm{H}, t-\mathrm{Bu}), 1.39\left(\mathrm{t},{ }^{3} J=7.1,3 \mathrm{H}, \mathrm{H}-13\right), 1.80-1.93(\mathrm{~m}, 2 \mathrm{H}$, $\mathrm{H}-7), 2.20\left(\mathrm{~s}, 3 \mathrm{H}, \mathrm{CH}_{3}\right), 2.22-2.31(\mathrm{~m}, 2 \mathrm{H}, \mathrm{H}-8), 4.01(\mathrm{t}$, $\left.{ }^{3} J=6.5,2 \mathrm{H}, \mathrm{H}-6\right), 4.37\left(\mathrm{dq},{ }^{3} J=7.1,{ }^{2} J=1.1,2 \mathrm{H}, \mathrm{H}-12\right)$, 4.93-5.15 (m, 2H, H-10), 5.86 (ddt, ${ }^{3} J=17.0,{ }^{3} J=10.3$, $\left.{ }^{3} J=6.7,1 \mathrm{H}, \mathrm{H}-9\right), 8.44$ (s, 1H, CHN), 9.11 (br s, 1H, NH); ${ }^{13} \mathrm{C}$ NMR $\left(100 \mathrm{MHz}, \mathrm{CDCl}_{3}\right): \delta=7.4\left(+, \mathrm{CH}_{3}\right), 14.4(+, \mathrm{C}-$ 13), $22.6(+, t-\mathrm{Bu}), 29.3(-, \mathrm{C}-7), 30.1(-, \mathrm{C}-8), 57.9(+, t-$ $\mathrm{Bu}), 60.9$ (-, C-12), 74.7 (-, C-6), 115.0 (-, C-10), 116.2 $\left(\mathrm{C}_{\text {quat }}, \mathrm{C}-2\right), 120.3\left(\mathrm{C}_{\text {quat }}, \mathrm{C}-4\right), 126.2\left(\mathrm{C}_{\text {quat }}, \mathrm{C}-5\right), 138.0(+$, C-9), $149.6\left(\mathrm{C}_{\text {quat }}, \mathrm{C}-3\right), 150.3(+, \mathrm{CHN}), 160.1\left(\mathrm{C}_{\text {quat }}, \mathrm{C}-\right.$ 11); IR (KBr): $\tilde{\nu}\left[\mathrm{cm}^{-1}\right]=3437,3227,2980,2935,2869$, 1693, 1265, 1057, 1025, 746; MS (EI, $70 \mathrm{eV}): \mathrm{m} / \mathrm{z}$ $(\%)=368.2(6)\left[\mathrm{M}^{+\bullet}\right], 312.0(100)\left[\mathrm{M}-\mathrm{C}_{4} \mathrm{H}_{8}\right]^{+\bullet}$. Elemental analysis calcd (\%) for $\mathrm{C}_{18} \mathrm{H}_{28} \mathrm{~N}_{2} \mathrm{O}_{4} \mathrm{~S}$ (368.49): C 58.67, $\mathrm{H}$ 7.66, N 7.60; found: C 58.61, H 7.68, N 7.31; Crystal data: $\mathrm{C}_{18} \mathrm{H}_{28} \mathrm{~N}_{2} \mathrm{O}_{4} \mathrm{~S}, M_{\mathrm{r}}=368.49$, colourless flat prism, triclinic, space group $P 1, \quad a=8.9068(9) \AA, \quad b=10.5174(12) \AA$, 
$c=11.9303(12) \AA, \quad \alpha=71.989(12)^{\circ}, \quad \beta=84.932(12)^{\circ}, \quad \gamma=$ 70.971(13) $)^{\circ}, Z=2, V=1004.7(2) \AA^{3}, D_{\mathrm{x}}=1.218 \mathrm{mg} / \mathrm{m}^{3}, \mu=$ $0.184 \mathrm{~mm}^{-1}, F(000)=396$, crystal size $0.44 \times 0.30 \times 0.12 \mathrm{~mm}$, $\theta$-range for data collections $2.35^{\circ}-26.82^{\circ}$, index ranges $-11 \leq h \leq 11,-13 \leq k \leq 13,-15 \leq l \leq 15$, reflections collected/ unique $10,549 / 7803\left[R_{\mathrm{int}}=0.0218\right]$, data/restraints/parameters 7803/3/461, goodness-of-fit on $F^{2} 0.993$, final $R$ indices [I $>2 \sigma(\mathrm{I})] \quad R_{1}=0.0278, \quad w R_{2}=0.0654, R$ indices (all data) $R_{1}=0.0307, w R_{2}=0.0663$, largest diff. peak and hole 0.328

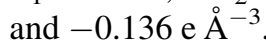

\subsection{3. (R)-Ethyl 3-(hex-5-enyloxy)-4-methyl-5-((2-methyl-} propan-2-ylsulfinamido)methyl)-1H-pyrrole-2-carboxylate (18)

Compound 14 (240 mg, $0.86 \mathrm{mmol})$, (R)-tert-butanesulfinamide $(\mathbf{1 5}, 125 \mathrm{mg}, 1.03 \mathrm{mmol})$ and $\mathrm{Ti}(\mathrm{OEt})_{4}(392 \mathrm{mg}$, $1.72 \mathrm{mmol})$ in dry dichloromethane were allowed to react according to the GP1 at room temperature for $36 \mathrm{~h}$ yielding $240 \mathrm{mg}$ (92\%, conversion corrected yield, $48 \mathrm{mg}$ of $14 \mathrm{re}-$ gained) of $18\left(\mathrm{Et}_{2} \mathrm{O} /\right.$ hexanes $\left.=1: 1 ; R_{f}=0.22\right)$ as white crystals, $\mathrm{mp}=75^{\circ} \mathrm{C}$; ${ }^{1} \mathrm{H}$ NMR $\left(300 \mathrm{MHz}, \mathrm{CDCl}_{3}\right): \delta=1.26(\mathrm{~s}, 9 \mathrm{H}$, $t-\mathrm{Bu}), 1.40\left(\mathrm{t},{ }^{3} J=7.1,3 \mathrm{H}, \mathrm{H}-14\right), 1.57-1.62$ (m, 2H, H-8), 1.77-1.83 (m, 2H, H-7), 2.10-2.15 (m, 2H, H-9), 2.21 (s, $\left.3 \mathrm{H}, \mathrm{CH}_{3}\right), 4.01\left(\mathrm{t},{ }^{3} J=6.5,2 \mathrm{H}, \mathrm{H}-6\right), 4.39\left(\mathrm{q},{ }^{3} J=7.1,2 \mathrm{H}\right.$, $\mathrm{H}-13)$, 4.96-5.07 (m, 2H, H-11), 5.83-5.85 (m, 1H, H-10), 8.46 (s, 1H, CHN), 9.25 (br s, $1 \mathrm{H}, \mathrm{NH}) ;{ }^{13} \mathrm{C} \mathrm{NMR}(75 \mathrm{MHz}$, $\left.\mathrm{CDCl}_{3}\right): \delta=6.7\left(+, \mathrm{CH}_{3}\right), 13.7(+, \mathrm{C}-14), 21.8(+, t-\mathrm{Bu}), 24.6$ (-, C-8), 28.9 (-, C-7), 32.8 (-, C-9), 57.1 (+, $t$-Bu), 60.2 (-, C-13), 74.5 (-, C-6), 114.2 (-, C-11), 115.5 (C quat $_{1}$ C-2), $119.6\left(\mathrm{C}_{\text {quat }}, \mathrm{C}-4\right), 125.5\left(\mathrm{C}_{\text {quat }}, \mathrm{C}-5\right), 137.8$ (+, C-10), 149.0 $\left(\mathrm{C}_{\text {quat }}, \mathrm{C}-3\right), 149.7$ (+, CHN), $159.4\left(\mathrm{C}_{\text {quat }}, \mathrm{C}-12\right)$; IR $(\mathrm{KBr})$ : $\tilde{\nu}\left[\mathrm{cm}^{-1}\right]=3445,3120,2989,2939,2866,2701,1693,1504$, 1267, 1056, 747; MS (FAB+): $m / z(\%)=383(100)[\mathrm{MH}]^{+}$, 326 (40) $\left[\mathrm{M}^{+}-\mathrm{C}_{4} \mathrm{H}_{8}\right]^{+}$; HRMS calcd for $\mathrm{C}_{19} \mathrm{H}_{31} \mathrm{~N}_{2} \mathrm{O}_{4} \mathrm{~S}^{+}$: 382.2015; found: $383.2005+2.8 \mathrm{ppm}$.

\subsection{General procedure (GP2) for the Grignard addition to $N$-tert-butanesulfinyl imines}

To a solution of the sulfinyl imine (1 equiv) in THF, the appropriate Grignard reagent $\left(2.5-3.3\right.$ equiv) in dry $\mathrm{Et}_{2} \mathrm{O}(5 \mathrm{~mL})$ was added dropwise and the conversion was monitored by TLC. The reaction was stirred at $50-60{ }^{\circ} \mathrm{C}$ for approximately $4 \mathrm{~h}$. Upon reaction completion, the excess organometallic reagent was destroyed with saturated aqueous $\mathrm{NH}_{4} \mathrm{Cl}(10 \mathrm{~mL})$ and the resulting suspension was diluted with brine $(10 \mathrm{~mL})$. The suspension was filtered through a plug of Celite and the filter cake was washed with EtOAc $(2 \times 10 \mathrm{~mL})$. The filtrate was transferred into a separatory funnel, the aqueous layer was washed with EtOAc $(3 \times 10 \mathrm{~mL})$, organic layers were combined, dried over $\mathrm{MgSO}_{4}$ and concentrated to afford the crude product. Diastereomeric ratios were determined by HPLC analyses or by NMR of the crude product. Purification was performed by flash-silica chromatography using Biotage SP4 chromatography system. Yields correspond to the mass balance of purified material.
3.3.1. (R)-Ethyl 3-(but-3-enyloxy)-5-(1-(1,1-dimethylethylsulfinamido)pent-4-enyl)-4-methyl-1H-pyrrole-2-

carboxylate (19)

Sulfinyl imine 16 (44 mg, $0.12 \mathrm{mmol}$ ) and but-3-enylmagnesium bromide $\left(0.24 \mathrm{~mL}, 1.3 \mathrm{M}\right.$ in $\left.\mathrm{Et}_{2} \mathrm{O}, 0.3 \mathrm{mmol}\right)$ were reacted according to the GP2. Flash-silica chromatography (Biotage SP4 chromatography system, EtOAc/cyclohexane= $7: 3)$ gave $30 \mathrm{mg}(61 \%)$ of $\mathbf{1 9}$ in a diastereomeric syn/anti ratio of 91:9. The diastereomeric ratio was determined by ${ }^{1} \mathrm{H}$ NMR. ${ }^{1} \mathrm{H}$ NMR $\left(300 \mathrm{MHz}, \mathrm{CDCl}_{3}\right): \delta=1.18\left(\mathrm{~s}, 9 \mathrm{H},\left(\mathrm{CH}_{3}\right)_{3}\right), 1.31(\mathrm{t}$, $\left.{ }^{3} J=7.1,3 \mathrm{H}, \mathrm{H}-12\right), 1.80-2.06$ (m, 4H, H-14, H-15), 1.90 (s, $\left.3 \mathrm{H}, \mathrm{CH}_{3}\right), 2.40-2.52$ (m, 2H, H-7), 3.95-4.06 (m, 2H, H6), 4.20-4.32 (m, 2H, H-11), 4.41-4.50 (m, 1H, H-13), 4.90-5.16 (m, 4H, H-9, H-17), 5.71 (dddd, ${ }^{3} J=16.9,{ }^{3} J=$ $\left.10.3,{ }^{3} J=6.5,{ }^{3} J=6.1,1 \mathrm{H}, \mathrm{H}-8\right), 5.80-5.96$ (m, $\left.1 \mathrm{H}, \mathrm{H}-16\right)$, 9.04 (br s, $1 \mathrm{H}, \mathrm{N}-\mathrm{H})$.

\subsection{2. (R)-Ethyl 3-(but-3-enyloxy)-5-(1-(1,1-dimethylethyl- sulfinamido)hex-5-enyl)-4-methyl-1H-pyrrole-2-carboxylate (20)}

Sulfinyl imine 16 (32 mg, $0.09 \mathrm{mmol}$ ) and pent-4-enylmagnesium bromide $\left(0.25 \mathrm{~mL}, 0.94 \mathrm{M}^{\text {in }} \mathrm{Et}_{2} \mathrm{O}, 0.23 \mathrm{mmol}\right)$ were reacted according to the GP2. Flash-silica chromatography (Biotage SP4 chromatography system, EtOAc/cyclohexane=7:3) gave $24 \mathrm{mg}(63 \%)$ of $\mathbf{2 0}$ in a diastereomeric syn/anti ratio of 93:7. The diastereomeric ratio was determined by ${ }^{1} \mathrm{H}$ NMR. ${ }^{1} \mathrm{H}$ NMR $\left(400 \mathrm{MHz}, \mathrm{CDCl}_{3}\right): \delta=1.14\left(\mathrm{~s}, 9 \mathrm{H},\left(\mathrm{CH}_{3}\right)_{3}\right), 1.29\left(\mathrm{t},{ }^{3} J=7.1,3 \mathrm{H}\right.$, $\mathrm{H}-12), 1.32-1.38$ (m, 2H, H-15), 1.88 (s, 3H, $\left.\mathrm{CH}_{3}\right), 1.92-2.01$ (m, 2H, H-16), 2.40-2.48 (m, 2H, H-7), 3.99 (dt, ${ }^{3} J=6.7$, $\left.{ }^{2} J=2.3,2 \mathrm{H}, \mathrm{H}-6\right), 4.16-4.29$ (m, 2H, H-11), 4.40 (ddd, ${ }^{3} J=7.3$, $\left.{ }^{3} J=7.3, J=1.6,1 \mathrm{H}, \mathrm{H}-13\right), 4.83-5.24$ (m, 4H, H-9, H-18), 5.65 (dddd, ${ }^{3} J=17.0,{ }^{3} J=10.1,{ }^{3} J=6.8,{ }^{3} J=6.7,1 H, H-8$ ), 5.85 (dddd, ${ }^{3} J=17.1,{ }^{3} J=10.3,{ }^{3} J=6.8,{ }^{3} J=6.7,1 \mathrm{H}, \mathrm{H}-17$ ), 9.01 (br s, $1 \mathrm{H}$, $\mathrm{N}-\mathrm{H}) ;{ }^{13} \mathrm{C} \mathrm{NMR}\left(100 \mathrm{MHz}, \mathrm{CDCl}_{3}\right): \delta=7.5\left(+, \mathrm{CH}_{3}\right), 14.5(+$, C-12), $22.6\left(+,\left(\mathrm{CH}_{3}\right)_{3}\right), 25.2(-, \mathrm{C}-15), 33.2(-, \mathrm{C}-14), 34.6$ $(-, \mathrm{C}-16), 35.5(-, \mathrm{C}-7), 52.1(+, \mathrm{C}-13), 55.7\left(\mathrm{C}_{\text {quat }}, \mathrm{C}-\right.$ $\left.\left(\mathrm{CH}_{3}\right)_{3}\right), 60.0(-, \mathrm{C}-11), 74.1(-, \mathrm{C}-6), 110.5\left(\mathrm{C}_{\text {quat }}, \mathrm{C}-2\right), 111.0$ ( $\left.\mathrm{C}_{\text {quat }}, \mathrm{C}-4\right), 115.2(-, \mathrm{C}-18), 116.6(-, \mathrm{C}-9), 131.5\left(\mathrm{C}_{\text {quat }}, \mathrm{C}-5\right)$, 134.9 (+, C-8), 137.9 (+, C-17), 149.8 (C quart, C-3), $160.4\left(\mathrm{C}_{\text {quat }}\right.$, $\mathrm{C}-11)$; IR (neat): $\tilde{\nu}\left[\mathrm{cm}^{-1}\right]=3448,3255,3077,2979,2929,2866$, $1665,1468,1032,911 ; \mathrm{MS}(\mathrm{FAB}+): \mathrm{m} / z(\%)=425(23)[\mathrm{M}+\mathrm{H}]^{+}$, 304 (100) $\left[\mathrm{M}^{+}-\mathrm{NH}-(\mathrm{S}=\mathrm{O})\left(\mathrm{CH}_{3}\right)_{3}\right]^{+} ;$HRMS calcd for $\mathrm{C}_{22} \mathrm{H}_{37} \mathrm{O}_{4} \mathrm{~N}_{2} \mathrm{~S}$ : 425.2574; found: $425.2487+3.1 \mathrm{ppm}$.

\subsection{3. (R)-Ethyl 3-(but-3-enyloxy)-5-(1-(1,1-dimethylethyl- sulfinamido)hept-6-enyl)-4-methyl-1H-pyrrole-2- carboxylate (21)}

Sulfinyl imine 16 (40 mg, $0.11 \mathrm{mmol})$ and hex-5-enylmagnesium bromide $\left(0.34 \mathrm{~mL}, 0.82 \mathrm{M}^{\text {in }} \mathrm{Et}_{2} \mathrm{O}, 0.28 \mathrm{mmol}\right)$ were reacted according to the GP2. Flash-silica chromatography (Biotage SP4 chromatography system, EtOAc/cyclohexane=7:3) gave $31 \mathrm{mg}(64 \%)$ of $\mathbf{2 1}$ in a diastereomeric syn/anti ratio of 91:9. The diastereomeric ratio was determined by ${ }^{1} \mathrm{H}$ NMR. ${ }^{1} \mathrm{H}$ NMR (400 MHz, $\mathrm{CDCl}_{3}$ ): $\delta=1.11-1.36$ (m, 4H, H-15, H-16), 1.15 (s, $\left.9 \mathrm{H},\left(\mathrm{CH}_{3}\right)_{3}\right), 1.29\left(\mathrm{t},{ }^{3} \mathrm{~J}=7.1,3 \mathrm{H}, \mathrm{H}-12\right), 1.67-1.83(\mathrm{~m}, 2 \mathrm{H}, \mathrm{H}-$ 14), 1.88 (s, 3H, $\mathrm{CH}_{3}$ ), 1.90-1.99 (m, 2H, H-17), 2.44 (ddt, $\left.{ }^{3} J=6.8,{ }^{3} J=6.8,{ }^{2} J=1.4,2 \mathrm{H}, \mathrm{H}-7\right), 3.50\left(\mathrm{~d},{ }^{3} J=2.2,1 \mathrm{H}, \mathrm{N}-\mathrm{H}\right)$, 
3.94-4.05 (m, 2H, H-69), 4.17-4.30 (m, 2H, H-11), 4.35-4.44 (m, 1H, H-13), 4.83-4.93 (m, 2H, H-9), 4.98-5.12 (m, 2H, H19), 5.67 (dddd, ${ }^{3} J=17.0,{ }^{3} J=10.2,{ }^{3} J=6.7,{ }^{3} J=6.7,1 \mathrm{H}, \mathrm{H}-8$ ), 5.86 (dddd, ${ }^{3} J=17.1,{ }^{3} J=10.3,{ }^{3} J=6.7,{ }^{3} J=6.7,1 \mathrm{H}, \mathrm{H}-18$ ), 9.00 (br s, $1 \mathrm{H}, \mathrm{H}$-pyrrole); ${ }^{13} \mathrm{C} \mathrm{NMR}\left(100 \mathrm{MHz}, \mathrm{CDCl}_{3}\right): \delta=7.5(+$, $\left.\mathrm{CH}_{3}\right), 14.5$ (+, C-12), $22.6\left(+,\left(\mathrm{CH}_{3}\right)_{3}\right), 25.4(-, \mathrm{C}-16), 28.4$ (-, C-15), 33.4 (-, C-14), 34.6 (-, C-17), 35.9 (-, C-7), 52.1 $(+, \mathrm{C}-13), 55.7\left(\mathrm{C}_{\text {quat }}, \mathrm{C}-\left(\mathrm{CH}_{3}\right)_{3}\right), 59.9(-, \mathrm{C}-11), 74.1(-, \mathrm{C}-$ 6), $110.5\left(\mathrm{C}_{\text {quat }}, \mathrm{C}-2\right), 111.0\left(\mathrm{C}_{\text {quat }}, \mathrm{C}-4\right), 114.7$ (-, C-19), 116.6 (-, C-9), $131.6\left(\mathrm{C}_{\text {quat }}, \mathrm{C}-5\right), 135.0$ (+, C-8), 138.4 (+, C-18), $149.8 \quad\left(\mathrm{C}_{\text {quart }}, \mathrm{C}-3\right), \quad 160.4 \quad\left(\mathrm{C}_{\text {quat }}, \mathrm{C}-11\right) ; \quad \mathrm{IR}$ (neat): $\tilde{\nu}$ $\left[\mathrm{cm}^{-1}\right]=3422,2979,2929,2861,1647,1465,1280,1032 ; \mathrm{MS}$ $(\mathrm{EI}, \quad 70 \mathrm{eV}): \quad \mathrm{m} / \mathrm{z} \quad(\%)=438.2 \quad(6) \quad\left[\mathrm{M}^{+*}\right], \quad 318.2 \quad(100)$ $\left[\mathrm{M}^{+\cdot}-{ }^{-} \mathrm{NBoc}-(\mathrm{S}=\mathrm{O})\left(\mathrm{CH}_{3}\right)_{3}\right]$; HRMS calcd for $\mathrm{C}_{23} \mathrm{H}_{38} \mathrm{~N}_{2} \mathrm{O}_{4} \mathrm{~S}$ $\left[\mathrm{M}^{+\bullet}\right]$ : 438.2548; found: $438.2548 \pm 1.9 \mathrm{ppm}$.

\subsection{4. (R)-Ethyl 5-(1-(1,1-dimethylethylsulfinamido)pent- 4-enyl)-4-methyl-3-(pent-4-enyloxy)-1H-pyrrole-2- carboxylate (22)}

Sulfinyl imine 17 (50 mg, $0.14 \mathrm{mmol})$ and but-3-enylmagnesium bromide $\left(0.28 \mathrm{~mL}, 1.3 \mathrm{M}\right.$ in $\left.\mathrm{Et}_{2} \mathrm{O}, 0.35 \mathrm{mmol}\right)$ were reacted according to the GP2. Flash-silica chromatography (Biotage SP4 chromatography system, EtOAc/cyclohexane= $7: 3)$ gave $40 \mathrm{mg}(68 \%)$ of $\mathbf{2 2}$ in a diastereomeric syn/anti ratio of 90:7. The diastereomeric ratio was determined by ${ }^{1} \mathrm{H}$ NMR and HPLC. ${ }^{1} \mathrm{H}$ NMR $\left(600 \mathrm{MHz}, \mathrm{CDCl}_{3}\right): \delta=1.24(\mathrm{~s}, 9 \mathrm{H}$, $\left.\left(\mathrm{CH}_{3}\right)_{3}\right), 1.37\left(\mathrm{t},{ }^{3} J=7.1,3 \mathrm{H}, \mathrm{H}-13\right), 1.84-1.89(\mathrm{~m}, 2 \mathrm{H}, \mathrm{H}-$ 7), $1.97\left(\mathrm{~s}, 3 \mathrm{H}, \mathrm{CH}_{3}\right), 1.09-2.03(\mathrm{~m}, 2 \mathrm{H}, \mathrm{H}-15), 2.04-2.12$ (m, 2H, H-16), 2.24-2.29 (m, 2H, H-8), $3.55\left(\mathrm{~d},{ }^{3} J=1.5,1 \mathrm{H}\right.$, $\mathrm{N}-\mathrm{H}), 4.04\left(\mathrm{dt},{ }^{2} J=6.4,{ }^{3} J=2.7,2 \mathrm{H}, \mathrm{H}-6\right), 4.30-4.37$ (m, 2H, $\mathrm{H}-12), 4.53$ (dt, $\left.{ }^{3} J=7.1,{ }^{3} J=1.9,1 \mathrm{H}, \mathrm{H}-14\right), 5.00$ (ddt, ${ }^{3} J=10.3,{ }^{2} J^{\dagger}$ and ${ }^{4} J,{ }^{\dagger} 1 \mathrm{H}, \mathrm{H}-10 \mathrm{cis}$ ), 5.03 (ddt, ${ }^{3} J=10.3,{ }^{2} J^{\dagger}$ and ${ }^{4} J,{ }^{\dagger} 1 \mathrm{H}, \mathrm{H}-18 \mathrm{cis}$ ), 5.05 (ddt, ${ }^{3} J=16.9,{ }^{2} J^{\dagger}$ and ${ }^{4} J,{ }^{\dagger} 1 \mathrm{H}$, H-18trans), 5.07 (ddt, ${ }^{3} J=17.0,{ }^{2} J$ and ${ }^{4} J{ }^{\dagger} 1 \mathrm{H}, \mathrm{H}-10$ trans), 5.78 (ddt, ${ }^{3} J=16.9,{ }^{3} J=10.3,{ }^{3} J=6.6,1 \mathrm{H}, \mathrm{H}-17$ ), 5.88 (ddt, $\left.{ }^{3} J=17.0,{ }^{3} J=10.3,{ }^{3} J=6.7,1 \mathrm{H}, \mathrm{H}-19\right), 8.98$ (br s, $1 \mathrm{H}$, pyrrole-H); ${ }^{13} \mathrm{C}$ NMR $\left(150 \mathrm{MHz}, \mathrm{CDCl}_{3}\right): \delta=7.5\left(+, \mathrm{CH}_{3}\right), 14.5$ (+, C-13), $22.6\left(+,\left(\mathrm{CH}_{3}\right)_{3}\right), 29.4(-, \mathrm{C}-7), 30.1(-, \mathrm{C}-16)$, 30.2 (-, C-8), 35.0 (-, C-15), $51.6(+, \mathrm{C}-14), 55.7\left(\mathrm{C}_{\text {quat }}\right.$, $\left.\mathrm{C}-\left(\mathrm{CH}_{3}\right)_{3}\right), 59.9(-, \mathrm{C}-12), 74.3(-, \mathrm{C}-6), 110.6\left(\mathrm{C}_{\text {quat }}, \mathrm{C}-2\right)$, 111.1 (C $\left._{\text {quat }}, \mathrm{C}-4\right), 114.8$ (-, C-10), 115.9 (, C-18), 131.1 $\left(\mathrm{C}_{\text {quat }}, \mathrm{C}-5\right), 137.0(+, \mathrm{C}-17), 138.2(+, \mathrm{C}-9), 150.0\left(\mathrm{C}_{\text {quat }}, \mathrm{C}-\right.$ $3), \quad 160.3 \quad\left(\mathrm{C}_{\text {quat }}, \quad \mathrm{C}-11\right) ; \quad \mathrm{IR} \quad$ (neat): $\tilde{\nu} \quad\left[\mathrm{cm}^{-1}\right]=3460$, 3252, 3077, 2978, 2927, 2868, 1665, 1468, 1032, 911; MS $(\mathrm{EI}, \quad 70 \mathrm{eV}): \quad \mathrm{m} / \mathrm{z} \quad(\%)=424.2 \quad(6) \quad\left[\mathrm{M}^{+*}\right], \quad 304.2 \quad(100)$ $\left[\mathrm{M}^{+\cdot}-{ }^{-} \mathrm{NH}-(\mathrm{S}=\mathrm{O})\left(\mathrm{CH}_{3}\right)_{3}\right], \quad 367.8 \quad\left[\mathrm{M}^{+\cdot}-{ }^{\cdot} \mathrm{C}_{4} \mathrm{H}_{9}\right] ; \quad$ HRMS calcd for $\mathrm{C}_{22} \mathrm{H}_{37} \mathrm{~N}_{2} \mathrm{O}_{4} \mathrm{~S}\left[\mathrm{MH}^{+}\right.$]: 425.2474 ; found: $425.2475 \pm$ $0.3 \mathrm{ppm}$.

\subsection{5. (R)-Ethyl 5-(1-(1,1-dimethylethylsulfinamido)hex- \\ 5-enyl)-4-methyl-3-(pent-4-enyloxy)-1H-pyrrole-2- carboxylate (23)}

Sulfinyl imine $17(100 \mathrm{mg}, 0.27 \mathrm{mmol})$ and pent-4-enylmagnesium bromide $\left(0.73 \mathrm{~mL}, 1.3 \mathrm{M}\right.$ in $\left.\mathrm{Et}_{2} \mathrm{O}, 0.90 \mathrm{mmol}\right)$

\footnotetext{
${ }^{\dagger}$ Coupling constants smaller than $2 \mathrm{~Hz}$ are not precise and are therefore not documented.
}

were reacted according to the GP2. Flash-silica chromatography (Biotage SP4 chromatography system, EtOAc/cyclohexane $=7: 3)$ gave $40 \mathrm{mg}(71 \%)$ of $\mathbf{2 3}$ in a diastereomeric synlanti ratio of 9:1. The diastereomeric ratio was determined by ${ }^{1} \mathrm{H}$ NMR and HPLC. ${ }^{1} \mathrm{H}$ NMR $\left(600 \mathrm{MHz}, \mathrm{CDCl}_{3}\right): \delta=1.20$ $\left(\mathrm{s}, 9 \mathrm{H},\left(\mathrm{CH}_{3}\right)_{3}\right), 1.35\left(\mathrm{t},{ }^{3} \mathrm{~J}=7.0,3 \mathrm{H}, \mathrm{H}-13\right), 1.22-1.46(\mathrm{~m}, 2 \mathrm{H}$, $\mathrm{H}-16), 1.77-1.93$ (m, 4H, H-7, H-15), $1.95\left(\mathrm{~s}, 3 \mathrm{H}, \mathrm{CH}_{3}\right)$, 1.98-2.07 (m, 2H, H-17), 2.22-2.29 (m, 2H, H-8), 4.03 (m, $3 \mathrm{H}, \mathrm{H}-6, \mathrm{~N}-\mathrm{H}), 4.37$ (m, 2H, H-12), 4.43-4.48 (m, 1H, H14), $4.90-5.10(\mathrm{~m}, 4 \mathrm{H}, \mathrm{H}-10, \mathrm{H}-19), 5.67-5.75(\mathrm{~m}, 1 \mathrm{H}, \mathrm{H}-$ 18), 5.80-5.90 (m, 1H, H-9), $9.30(\mathrm{~s}, 1 \mathrm{H}, \mathrm{N}-\mathrm{H}) ;{ }^{13} \mathrm{C}$ NMR $\left(150 \mathrm{MHz}, \mathrm{CDCl}_{3}\right): \delta=7.5\left(+, \mathrm{CH}_{3}\right), 14.5(+, \mathrm{C}-13), 22.6$ $\left(+,\left(\mathrm{CH}_{3}\right)_{3}\right), 25.2(-, \mathrm{C}-16), 29.4(-, \mathrm{C}-7), 30.2(-, \mathrm{C}-8)$, 33.2 (-, C-17), 35.7 (-, C-15), 52.4 (+, C-14), 55.8 ( $\mathrm{C}_{\text {quat }}$, $\left.\mathrm{C}-\left(\mathrm{CH}_{3}\right)_{3}\right), 60.0(-, \mathrm{C}-12), 74.3(-, \mathrm{C}-6), 110.4\left(\mathrm{C}_{\text {quat }}, \mathrm{C}-2\right)$, 110.7 ( $\left.\mathrm{C}_{\text {quat }}, \mathrm{C}-4\right), 114.8$ (-, C-10), 115.1 (-, C-19), 132.0 $\left(\mathrm{C}_{\text {quat }}, \mathrm{C}-5\right), 137.9$ (+, C-18), $138.2(+, \mathrm{C}-9), 149.9\left(\mathrm{C}_{\text {quart }}\right.$, $\mathrm{C}-3), \quad 160.6 \quad\left(\mathrm{C}_{\text {quat }}, \mathrm{C}-11\right)$; IR (neat): $\tilde{\nu} \quad\left[\mathrm{cm}^{-1}\right]=3458$, 3252, 3075, 2976, 2928, 2866, 1665, 1468, 1273, 1032; MS (EI, $70 \mathrm{eV}): \quad \mathrm{m} / \mathrm{z} \quad(\%)=438.2 \quad$ (6) $\quad\left[\mathrm{M}^{+\cdot}\right], \quad 318.1 \quad(100)$ $\left[\mathrm{M}^{+\cdot}-{ }^{-} \mathrm{NH}-(\mathrm{S}=\mathrm{O})\left(\mathrm{CH}_{3}\right)_{3}\right]$; HRMS calcd for $\mathrm{C}_{23} \mathrm{H}_{38} \mathrm{~N}_{2} \mathrm{O}_{4} \mathrm{~S}$ $\left[\mathrm{M}^{+\bullet}\right]$ : 438.2555; found: $438.2550 \pm 0.7 \mathrm{ppm}$.

\subsection{6. (R)-Ethyl 5-(1-(1,1-dimethylethylsulfinamido)hept- 6-enyl)-4-methyl-3-(pent-4-enyloxy)-1H-pyrrole-2- \\ carboxylate (24)}

Sulfinyl imine 17 (200 mg, $0.54 \mathrm{mmol})$ and hex-5-enylmagnesium bromide $\left(1.27 \mathrm{~mL}, 0.94 \mathrm{M}\right.$ in $\left.\mathrm{Et}_{2} \mathrm{O}, 1.19 \mathrm{mmol}\right)$ were reacted according to the GP2. Flash-silica chromatography (Biotage SP4 chromatography system, EtOAc/cyclohexane=7:3) gave $159 \mathrm{mg}(65 \%)$ of $\mathbf{2 4}$ in a diastereomeric syn/anti ratio of 93:7. The diastereomeric ratio was determined by ${ }^{1} \mathrm{H}$ NMR. ${ }^{1} \mathrm{H}$ NMR $\left(600 \mathrm{MHz}, \mathrm{CDCl}_{3}\right): \delta=1.21$ (s, 9H, $\left.\left(\mathrm{CH}_{3}\right)_{3}\right), 1.22-1.41(\mathrm{~m}, 7 \mathrm{H}, \mathrm{H}-13, \mathrm{H}-16, \mathrm{H}-17), 1.77-1.91$ (m, 4H, H-7, H-15), 1.95 (s, 3H, CH $\left.{ }_{3}\right), 1.98-2.04(\mathrm{~m}, 2 \mathrm{H}$, $\mathrm{H}-18), 2.21-2.32(\mathrm{~m}, 2 \mathrm{H}, \mathrm{H}-8), 3.77-3.88(\mathrm{~m}, 1 \mathrm{H}, \mathrm{N}-\mathrm{H})$, 3.94-4.05 (m, 2H, H-6), 4.23-4.38 (m, 2H, H-12), 4.414.51 (m, 1H, H-14), 4.88-5.09 (m, 4H, H-10, H-20), 5.695.78 (m, 1H, H-19), 5.82-5.92 (m, 1H, H-9), 9.23 (br s, $1 \mathrm{H}$, $\mathrm{N}-\mathrm{H}) ;{ }^{13} \mathrm{C}$ NMR $\left(150 \mathrm{MHz}, \mathrm{CDCl}_{3}\right): \delta=7.5\left(+, \mathrm{CH}_{3}\right), 14.5$ (+, C-13), $22.6\left(+,\left(\mathrm{CH}_{3}\right)_{3}\right), 25.4(-, \mathrm{C}-17), 28.4(-, \mathrm{C}-16)$, 29.4 (-, C-7), 30.2 (-, C-8), 33.4 (-, C-18), 36.1 (-, C15), $52.4(+, \mathrm{C}-14), 55.8\left(\mathrm{C}_{\text {quat }}, \mathrm{C}-\left(\mathrm{CH}_{3}\right)_{3}\right), 60.0(-, \mathrm{C}-12)$, 74.3 (-, C-6), $110.4\left(\mathrm{C}_{\text {quat }}, \mathrm{C}-2\right), 110.8$ (C $\left.\mathrm{C}_{\text {quat }}, \mathrm{C}-4\right), 114.6$ (-, C-10), 114.8 (, C-20), $132.0\left(\mathrm{C}_{\text {quat }}, \mathrm{C}-5\right), 138.2$ (+, C19), 138.4 (+, C-9), 149.9 ( $\left.\mathrm{C}_{\text {quart }}, \mathrm{C}-3\right), 160.6\left(\mathrm{C}_{\text {quat }}, \mathrm{C}-11\right)$; IR (neat): $\tilde{\nu}\left[\mathrm{cm}^{-1}\right]=3458,3252,3075,2976,2928,2866$, $1665,1469,1280,1033,910,733 ; \mathrm{MS}(\mathrm{FAB}+): \mathrm{m} / \mathrm{z}$ $(\%)=453(20)[\mathrm{MH}]^{+}, 332(100)\left[\mathrm{M}-\mathrm{NH}-(\mathrm{S}=\mathrm{O})\left(\mathrm{CH}_{3}\right)_{3}\right]^{+}$; HRMS calcd for $\mathrm{C}_{24} \mathrm{H}_{41} \mathrm{~N}_{2} \mathrm{O}_{4} \mathrm{~S}\left[\mathrm{M}^{+*}\right]$ : 453.2787 ; found: $453.2813+10 \mathrm{ppm}$.

\subsection{7. (R)-Ethyl 5-(1-(1,1-dimethylethylsulfinamido)pent-4-} enyl)-3-(hex-5-enyloxy)-4-methyl-1H-pyrrole-2-carboxylate (25)

Sulfinyl imine 18 (63 mg, $0.14 \mathrm{mmol})$ and but-3-enylmagnesium bromide $\left(0.33 \mathrm{~mL}, 1.3 \mathrm{M}\right.$ in $\left.\mathrm{Et}_{2} \mathrm{O}, 0.41 \mathrm{mmol}\right)$ were 
reacted according to the GP2. Flash-silica chromatography (Biotage SP4 chromatography system, EtOAc/cyclohexane=7:3) gave $48 \mathrm{mg}(68 \%)$ of $\mathbf{2 5}$ in a diastereomeric syn/anti ratio of 95:5. The diastereomeric ratio was determined by ${ }^{1} \mathrm{H}$ NMR. ${ }^{1} \mathrm{H}$ NMR $\left(400 \mathrm{MHz}, \mathrm{CDCl}_{3}\right): \delta=1.23(\mathrm{~s}, 9 \mathrm{H}$, $\left.\left(\mathrm{CH}_{3}\right)_{3}\right), 1.28\left(\mathrm{t},{ }^{3} \mathrm{~J}=7.1,3 \mathrm{H}, \mathrm{H}-14\right), 1.45-1.58(\mathrm{~m}, 2 \mathrm{H}, \mathrm{H}-$ 8), $1.62-1.75(\mathrm{~m}, 2 \mathrm{H}, \mathrm{H}-7), 1.78-2.01(\mathrm{~m}, 4 \mathrm{H}, \mathrm{H}-16$ and $\mathrm{H}-17), 1.88\left(\mathrm{~s}, 3 \mathrm{H}, \mathrm{CH}_{3}\right), 2.01-2.10(\mathrm{~m}, 2 \mathrm{H}, \mathrm{H}-9), 3.85(\mathrm{~d}$, $\left.{ }^{3} J=2.9,1 \mathrm{H}, \mathrm{N}-\mathrm{H}\right), 3.93\left(\mathrm{t},{ }^{3} J=6.82 \mathrm{H}, \mathrm{H}-6\right), 4.13-4.29$ (m, $2 \mathrm{H}, \mathrm{H}-13), 4.36-4.48$ (m, 2H, H-15), 4.75-5.07 (m, 4H, H11 and $\mathrm{H}-19), 5.54-5.91$ (m, 2H, H-10 and $\mathrm{H}-18), 8.15$ (br s, $1 \mathrm{H}$, pyrrole-H); ${ }^{13} \mathrm{C}$ NMR $\left(100 \mathrm{~Hz} \mathrm{CDCl}_{3}\right): \delta=7.5(+$, $\left.\mathrm{CH}_{3}\right), 14.5$ (+, C-14), $22.6\left(+,\left(\mathrm{CH}_{3}\right)_{3}\right), 25.3(-, \mathrm{C}-8), 29.7$ (-, C-7), 30.1 (-, C-17), $33.6(-, \mathrm{C}-9), 35.2(-, \mathrm{C}-16), 52.0$ $(+, \mathrm{C}-15), 55.8\left(\mathrm{C}_{\text {quat }}, \mathrm{C}-\left(\mathrm{CH}_{3}\right)_{3}\right), 60.0(-, \mathrm{C}-13), 74.8(-, \mathrm{C}-$ 6), $110.9\left(\mathrm{C}_{\text {quat }}, \mathrm{C}-2\right), 111.1\left(\mathrm{C}_{\text {quat }}, \mathrm{C}-4\right), 114.9(-, \mathrm{C}-11)$, 115.8 (-, C-19), $131.6\left(\mathrm{C}_{\text {quat }}, \mathrm{C}-5\right), 137.1$ (+, C-18), 138.7 (+, C-10), $150.0\left(\mathrm{C}_{\text {quat }}, \mathrm{C}-3\right), 160.6\left(\mathrm{C}_{\text {quat }}, \mathrm{C}-12\right)$; IR (neat): $\tilde{\nu}\left[\mathrm{cm}^{-1}\right]=3461,3300,2978,2933,2867,1663,1272,1031$, 909; MS (FAB+): $m / z \quad(\%)=439$ (26) $[\mathrm{MH}]^{+}, 318(100)$ $\left[\mathrm{M}-\mathrm{NH}-(\mathrm{S}=\mathrm{O})\left(\mathrm{CH}_{3}\right)_{3}\right]^{+}$; HRMS calcd for $\mathrm{C}_{23} \mathrm{H}_{39} \mathrm{O}_{4} \mathrm{O}_{2} \mathrm{~S}$ : 439.2631; found: $439.2614+3.9 \mathrm{ppm}$.

\subsection{8. (R)-Ethyl 5-(1-(1,1-dimethylethylsulfinamido)hex-5-} enyl)-3-(hex-5-enyloxy)-4-methyl-1H-pyrrole-2-carboxylate (26)

Sulfinyl imine 18 (63 mg, $0.14 \mathrm{mmol}$ ) and pent-4-enylmagnesium bromide $\left(0.44 \mathrm{~mL}, 0.94 \mathrm{M}^{\text {in }} \mathrm{Et}_{2} \mathrm{O}, 0.41 \mathrm{mmol}\right.$ ) were reacted according to the GP2. Flash-silica chromatography (Biotage SP4 chromatography system, EtOAc/cyclohexane=7:3) gave $36 \mathrm{mg}(50 \%)$ of $\mathbf{2 6}$ in a diastereomeric syn/anti ratio of 91:9. The diastereomeric ratio was determined by ${ }^{1} \mathrm{H}$ NMR. ${ }^{1} \mathrm{H}$ NMR $\left(400 \mathrm{MHz}, \mathrm{CDCl}_{3}\right): \delta=1.14$ (s, 9H, $\left.\left(\mathrm{CH}_{3}\right)_{3}\right), 1.18-1.41(\mathrm{~m}, 2 \mathrm{H}, \mathrm{H}-17), 1.28\left(\mathrm{t},{ }^{3} J=7.1,3 \mathrm{H}, \mathrm{H}-\right.$ 14), $1.46-1.57(\mathrm{~m}, 2 \mathrm{H}, \mathrm{H}-8), 1.62-1.84(\mathrm{~m}, 4 \mathrm{H}, \mathrm{H}-7$ and $\mathrm{H}-16), 1.88$ (s, 3H, $\left.\mathrm{CH}_{3}\right), 1.91-2.00$ (m, $\left.2 \mathrm{H}, \mathrm{H}-18\right), 2.01-$ $2.10(\mathrm{~m}, 2 \mathrm{H}, \mathrm{H}-9), 3.62\left(\mathrm{~d},{ }^{3} \mathrm{~J}=2.5,1 \mathrm{H}, \mathrm{N}-\mathrm{H}\right), 3.88-3.98$ (m, 2H, H-6), 4.15-4.29 (m, 2H, H-13), 4.36-4.45 (m, 1H, $\mathrm{H}-15), 4.85-5.99(\mathrm{~m}, 4 \mathrm{H}, \mathrm{H}-11$ and $\mathrm{H}-20), 5.59-5.82(\mathrm{~m}$, $2 \mathrm{H}, \mathrm{H}-10$ and $\mathrm{H}-19$ ), 9.03 (br s, $1 \mathrm{H}$, pyrrole-H); ${ }^{13} \mathrm{C}$ NMR $\left(100 \mathrm{MHz}, \mathrm{CDCl}_{3}\right): \delta=7.5\left(+, \mathrm{CH}_{3}\right), 14.5(+, \mathrm{C}-14), 22.6$ $\left(+,\left(\mathrm{CH}_{3}\right)_{3}\right), 25.2(-, \mathrm{C}-17), 25.3(-, \mathrm{C}-8), 29.7(-, \mathrm{C}-7)$, 33.2 (-, C-18), $33.6(-, \mathrm{C}-9), 35.6(-, \mathrm{C}-16), 52.2(+, \mathrm{C}-$ $15), 55.8\left(\mathrm{C}_{\text {quat }}, \mathrm{C}-\left(\mathrm{CH}_{3}\right)_{3}\right), 60.0(-, \mathrm{C}-13), 74.8(-, \mathrm{C}-6)$, $110.5\left(\mathrm{C}_{\text {quat }}, \mathrm{C}-2\right), 110.9\left(\mathrm{C}_{\text {quat }}, \mathrm{C}-4\right), 114.6(-, \mathrm{C}-11)$, 115.2 (-, C-20), 131.6 ( $\left.\mathrm{C}_{\text {quat }}, \mathrm{C}-5\right), 137.9$ (+, C-19), 138.7 (+, C-10), $150.0\left(\mathrm{C}_{\text {quat }}, \mathrm{C}-3\right), 160.5$ ( $\left.\mathrm{C}_{\text {quat }}, \mathrm{C}-12\right)$; IR (neat): $\tilde{\nu}\left[\mathrm{cm}^{-1}\right]=3420,3257,2978,2931,2864,1664,1468,1273$, 1032; MS $(\mathrm{FAB}+): \mathrm{m} / \mathrm{z}(\%)=453(20)[\mathrm{MH}]^{+}, 332(100)$ $\left[\mathrm{M}-\mathrm{NH}-(\mathrm{S}=\mathrm{O})\left(\mathrm{CH}_{3}\right)_{3}\right]^{+}$; HRMS calcd for $\mathrm{C}_{24} \mathrm{H}_{41} \mathrm{O}_{4} \mathrm{O}_{2} \mathrm{~S}$ : 453.2787; found: $453.2807+4.5 \mathrm{ppm}$.

\subsection{9. (R)-Ethyl 5-(1-(1,1-dimethylethylsulfinamido)hept-6-} enyl)-3-(hex-5-enyloxy)-4-methyl-1H-pyrrole-2-carboxylate (27)

Sulfinyl imine 18 (63 mg, $0.16 \mathrm{mmol})$ and hex-5-enylmagnesium bromide $\left(0.44 \mathrm{~mL}, 0.94 \mathrm{M}^{\text {in }} \mathrm{Et}_{2} \mathrm{O}, 0.41 \mathrm{mmol}\right.$ ) were reacted according to the GP2. Flash-silica chromatography (Biotage SP4 chromatography system, EtOAc/cyclohexane $=7: 3)$ gave $36 \mathrm{mg}$ (72\%) of $\mathbf{2 1}$ in a diastereomeric syn/anti ratio of 94:6. The diastereomeric ratio was determined by proton NMR. ${ }^{1} \mathrm{H}$ NMR $\left(400 \mathrm{MHz}, \mathrm{CDCl}_{3}\right): \delta=1.13(\mathrm{~s}, 9 \mathrm{H}$, $\left.\left(\mathrm{CH}_{3}\right)_{3}\right), 1.19-1.45(\mathrm{~m}, 4 \mathrm{H}, \mathrm{H}-17$ and 18$), 1.28\left(\mathrm{t},{ }^{3} \mathrm{~J}=7.1,3 \mathrm{H}\right.$, $\mathrm{H}-14), 1.46-1.58(\mathrm{~m}, 2 \mathrm{H}, \mathrm{H}-8), 1.60-1.84(\mathrm{~m}, 4 \mathrm{H}, \mathrm{H}-7$ and $\mathrm{H}-16), 1.88\left(\mathrm{~s}, 3 \mathrm{H}, \mathrm{CH}_{3}\right), 1.90-2.11(\mathrm{~m}, 4 \mathrm{H}, \mathrm{H}-9$ and $\mathrm{H}-$ 19), $3.72\left(\mathrm{~d},{ }^{3} J=2.9,1 \mathrm{H}, \mathrm{N}-\mathrm{H}\right), 3.86-3.98(\mathrm{~m}, 2 \mathrm{H}, \mathrm{H}-6)$, 4.16-4.31 (m, 2H, H-13), 4.33-4.43 (m, 1H, H-15), 4.80$5.01(\mathrm{~m}, 4 \mathrm{H}, \mathrm{H}-11$ and $\mathrm{H}-21), 5.59-5.83(\mathrm{~m}, 2 \mathrm{H}, \mathrm{H}-10$ and $\mathrm{H}-20), 9.17$ (br s, $1 \mathrm{H}$, pyrrole-H); ${ }^{13} \mathrm{C}$ NMR $(100 \mathrm{MHz}$, $\left.\mathrm{CDCl}_{3}\right): \delta=7.5\left(+, \mathrm{CH}_{3}\right), 14.5(+, \mathrm{C}-14), 22.6\left(+,\left(\mathrm{CH}_{3}\right)_{3}\right)$, 25.3 (-, C-8), 25.4 (-, C-18), 28.4 (-, C-17), 29.7 (-, C7), 33.4 (-, C-19), 33.7 (-, C-9), 36.1 (-, C-16), 52.3 (+, $\mathrm{C}-15), 55.8\left(\mathrm{C}_{\text {quat }}, \mathrm{C}-\left(\mathrm{CH}_{3}\right)_{3}\right), 59.9$ (-, C-13), 74.8 (-, C-6), $110.4\left(\mathrm{C}_{\text {quat }}, \mathrm{C}-2\right), 110.8\left(\mathrm{C}_{\text {quat }}, \mathrm{C}-4\right), 114.2(-, \mathrm{C}-11)$, 114.6 (-, C-21), 131.9 (C $\left.\mathrm{C}_{\text {quat }}, \mathrm{C}-5\right), 138.4$ (+, C-20), 138.7 (+, C-10), $150.0\left(\mathrm{C}_{\text {quat }}, \mathrm{C}-3\right), 160.6\left(\mathrm{C}_{\text {quat }}, \mathrm{C}-12\right)$; IR (neat): $\tilde{\nu}\left[\mathrm{cm}^{-1}\right]=3460,3256,3076,2977,2930,2861,1666,1469$, 1280, 1032, 994; MS $(\mathrm{FAB}+): \mathrm{m} / z(\%)=467(18)[\mathrm{MH}]^{+}$, 346 (100) $\left[\mathrm{M}-\mathrm{NH}-(\mathrm{S}=\mathrm{O})\left(\mathrm{CH}_{3}\right)_{3}\right]^{+}$; HRMS calcd for $\mathrm{C}_{25} \mathrm{H}_{42} \mathrm{O}_{4} \mathrm{~N}_{2} \mathrm{~S}$ : 466.2865 ; found: $466.2849+3.5 \mathrm{ppm}$.

3.4. (R)-1-tert-Butyl 2-ethyl 5-(1-(1,1-dimethylethylsulfinamido)hex-5-enyl)-4-methyl-3-(pent-4-enyloxy)-1H-pyrrole1,2-dicarboxylate 23-Boc and (R)-ethyl 5-(1(N-(tert-butoxycarbonyl)-2-methylpropan-2-ylsulfinamido)hex-5-enyl)-4-methyl-3-(pent-4-enyloxy)1H-pyrrole-2-carboxylate 23a-Boc

To a solution of $23(130 \mathrm{mg}, 0.30 \mathrm{mmol})$ in dry $\mathrm{CH}_{2} \mathrm{Cl}_{2}$ $(20 \mathrm{~mL})$ at room temperature DMAP $(40.3 \mathrm{mg}, 0.33 \mathrm{mmol})$ was added and the mixture was stirred for $10 \mathrm{~min}$ under dinitrogen atmosphere. (Boc) $)_{2} \mathrm{O}(131 \mathrm{mg}, 0.60 \mathrm{mmol})$ was added and the mixture was stirred overnight (Scheme 4). The solution was washed with water $(4 \times 10 \mathrm{~mL})$ and dried over $\mathrm{MgSO}_{4}$. The solvent was evaporated in vacuum and the two products were separated by column chromatography on silica gel (cyclohexane/EtOAc $=8: 2, R_{f}=0.13$ and 0.47 ) to give $44 \mathrm{mg}$ of 23-Boc (29\%) as a colourless oil and $99 \mathrm{mg}$ of 23a-Boc $(61 \%)$ as a colourless oil.

\subsubsection{Compound 23-Boc}

IR: $\tilde{\nu}\left[\mathrm{cm}^{-1}\right]=3450,3302,3077,2979,2934,2870,1713$; MS (EI, $70 \mathrm{eV}): m / z(\%)=538.1(0.2)\left[\mathrm{M}^{+*}\right], 318.2(100)$ $\left[\mathrm{M}^{+\cdot}-{ }^{-} \mathrm{NBoc}-(\mathrm{S}=\mathrm{O})\left(\mathrm{CH}_{3}\right)_{3}\right]$; HRMS calcd for $\mathrm{C}_{28} \mathrm{H}_{46} \mathrm{~N}_{2} \mathrm{O}_{6} \mathrm{~S}$ $\left[\mathrm{M}^{+\bullet}\right.$ ]: 538.3077; found: $538.3075 \pm 1.2 \mathrm{ppm}$.

\subsubsection{Compound 23a-Boc}

${ }^{1} \mathrm{H}$ NMR (600 MHz, $\left.\mathrm{CDCl}_{3}\right): \delta=1.15-1.23(\mathrm{~m}, 2 \mathrm{H}, \mathrm{H}-16)$, 1.27 (s, 9H, TBS), $1.34\left(\mathrm{t},{ }^{3} J=7.1,3 \mathrm{H}, \mathrm{H}-13\right), 1.52(\mathrm{~s}, 9 \mathrm{H}$, Boc), $1.69-1.79(\mathrm{~m}, 1 \mathrm{H}, \mathrm{H}-15 \mathrm{~A}), 1.80-1.87$ (m, 2H, H-7), 1.95-2.05 (m, 2H, H-17), 2.02 (s, 3H, $\left.\mathrm{CH}_{3}\right), 2.19-2.29$ (m, $2 \mathrm{H}, \mathrm{H}-8), 2.67-2.80$ (m, 1H, H-15B), 3.95-4.00 (m, 1H, H6A), 4.01-4.07 (m, 1H, H-6B), 4.23-4.36 (m, 2H, H-12), 4.57-4.65 (m, 1H, H-14), 4.89-5.11 (m, 4H, H-10, H-19), 


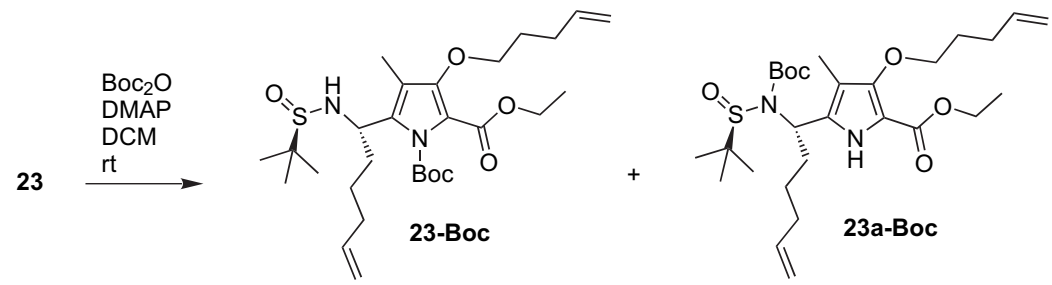

Scheme 4. Preparation of the Boc-protected diene 23.

5.71 (dddd, ${ }^{3} J=17.0,{ }^{3} J=10.3,{ }^{3} J=6.7,{ }^{3} J=6.7,1 \mathrm{H}, \mathrm{H}-18$ ), 5.86 (dddd, ${ }^{3} J=17.0,{ }^{3} J=10.3,{ }^{3} J=6.7,{ }^{3} J=6.7,1 \mathrm{H}, \quad \mathrm{H}-9$ ), $9.70\left(b r s, 1 \mathrm{H}, \mathrm{H}\right.$-pyrrole); ${ }^{13} \mathrm{C} \mathrm{NMR}\left(75 \mathrm{MHz}, \mathrm{CDCl}_{3}\right)$ : $\delta=7.4\left(+, \mathrm{CH}_{3}\right), 14.5(+, \mathrm{C}-13), 22.8(+, \mathrm{TBS}), 26.3(-, \mathrm{C}-16)$, 28.3 (+, Boc), 29.5 (-, C-7), $30.2(-$, C-8), $33.2(-$, C-17), 33.5 (-, C-15), 45.5 (+, C-14), 59.6 (-, C-12), 60.4 ( $\mathrm{C}_{\text {quat }}$, TBS), 74.2 (-, C-6), 83.7 ( $\left.\mathrm{C}_{\text {quat }}, \mathrm{Boc}\right), 110.3$ ( $\left.\mathrm{C}_{\text {quat }}, \mathrm{C}-2\right)$, 112.5 (C $\left.\mathrm{C}_{\text {quat }}, \mathrm{C}-4\right), 114.7$ (-, C-10), 114.9 (-, C-19), 130.8 $\left(\mathrm{C}_{\text {quat }}, \mathrm{C}-5\right), 137.9(+, \mathrm{C}-18), 138.4(+, \mathrm{C}-9), 149.2\left(\mathrm{C}_{\text {quart }}, \mathrm{C}-\right.$ 3), 154.5 ( $\mathrm{C}_{\text {quart }}, \mathrm{C}-\mathrm{Boc}$ amide), $160.6\left(\mathrm{C}_{\text {quat }}, \mathrm{C}-11\right)$; IR: $\tilde{\nu}$ $\left[\mathrm{cm}^{-1}\right]=3450,3397,3077,2978,2932,1694 ;$ MS (EI, $\left.70 \mathrm{eV}\right)$ : $\mathrm{m} / \mathrm{z}(\%)=538.1(7)\left[\mathrm{M}^{+*}\right], 482.1(13)\left[\mathrm{M}-\mathrm{C}_{4} \mathrm{H}_{8}\right]^{+\cdot}, 318.2(94)$ $\left[\mathrm{M}^{+\cdot}-{ }^{-} \mathrm{NH}-(\mathrm{S}=\mathrm{O})\left(\mathrm{CH}_{3}\right)_{3}\right], 276.1$ (100) $\left[\mathrm{M}^{+\cdot}-{ }^{-} \mathrm{NH}-(\mathrm{S}=\mathrm{O})\right.$ $\left(\mathrm{CH}_{3}\right)_{3}-\mathrm{C}_{2} \mathrm{H}_{2} \mathrm{O}$; HRMS calcd for $\mathrm{C}_{28} \mathrm{H}_{46} \mathrm{~N}_{2} \mathrm{O}_{6} \mathrm{~S}\left[\mathrm{M}^{+*}\right.$ : 538.3077; found: $538.3079 \pm 0.4 \mathrm{ppm}$.

\subsection{General procedure (GP3) for ring-closing metathesis of sulfinyl imine dialkenes}

Sulfinyl imine dienes were dissolved as a $0.0005 \mathrm{M}$ solution in anhydrous and degassed $\mathrm{CH}_{2} \mathrm{Cl}_{2}$ under an argon atmosphere. Bis(tricyclohexylphosphine) benzylidine ruthenium(IV) dichloride (Grubbs I catalyst) $(15 \mathrm{~mol} \%)$, dissolved in anhydrous and degassed $\mathrm{CH}_{2} \mathrm{Cl}_{2}(50 \mathrm{~mL})$, was added slowly over a period of $2 \mathrm{~h}$ by a syringe pump to the reaction mixture at $40{ }^{\circ} \mathrm{C}$, and stirred for additional $24 \mathrm{~h}$. Additional catalyst $(15 \mathrm{~mol} \%)$ was added and the mixture was refluxed for $1 \mathrm{~d}$. After 50 equiv of DMSO was added, the mixture was stirred at room temperature for $1 \mathrm{~d}$. Concentration under reduced pressure and filtration through a pad of silica afforded the crude product, which was purified by HPLC.

\subsubsection{Ethyl 11-((R)-1,1-dimethylethylsulfinamido)-15-} methyl-2-oxa-13 azabicyclo[10.2.1]pentadeca-

1(14),6,12(15)-triene-14-carboxylate (28)

Compound 23 ( $20 \mathrm{mg}, 0.05 \mathrm{mmol}$ ) was reacted following the GP3. Preparative HPLC afforded $7 \mathrm{mg}$ of the ansa-bridged $28(47 \%$, conversion corrected yield, $5 \mathrm{mg}$ of $\mathbf{2 3}$ regained) as an inseparable 9:1 E/Z mixture and $3.5 \mathrm{mg}$ of the larger macrocycle $29(12 \%)$ as a mixture of stereoisomers.

\subsubsection{Compound 28}

${ }^{1} \mathrm{H}$ NMR (600 MHz, $\mathrm{CDCl}_{3}$, DQF-COSY, HSQC, HMBC): $\delta=0.84-0.92(\mathrm{~m}, 1 \mathrm{H}, \mathrm{H}-8 \mathrm{~A}), 0.92-1.02(\mathrm{~m}, 1 \mathrm{H}, \mathrm{H}-11 \mathrm{~A})$, $1.26\left(\mathrm{~s}, 9 \mathrm{H},\left(\mathrm{CH}_{3}\right)_{3}\right), 1.38\left(\mathrm{t}, 3 \mathrm{H},{ }^{3} J=7.13, \mathrm{H}-16\right), 1.43-1.53$ $(\mathrm{m}, 2 \mathrm{H}, \mathrm{H}-7 \mathrm{~A}, \mathrm{H}-8 \mathrm{~B}), 1.55-1.63(\mathrm{~m}, 1 \mathrm{H}, \mathrm{H}-13 \mathrm{~A}), 1.65-$ $1.75(\mathrm{~m}, 2 \mathrm{H}, \mathrm{H}-11 \mathrm{~B}, \mathrm{H}-12 \mathrm{~A}), 2.00-2.13(\mathrm{~m}, 4 \mathrm{H}, \mathrm{H}-7 \mathrm{~B}$,
$\mathrm{CH}_{3}$ ), 2.15-2.21 (m, 2H, H-12B, H-13B), 3.62 (br s, 1H, N$\mathrm{H})$, 4.13-4.20 (m, 1H, H-6A), 4.24-4.34 (m, 2H, H-6B, H$15 \mathrm{~A}), 4.43-4.49(\mathrm{~m}, 1 \mathrm{H}, \mathrm{H}-15 \mathrm{~B}), 4.54\left(\mathrm{dd}, 1 \mathrm{H},{ }^{2} J=11.96\right.$, $\left.{ }^{3} J=5.26, \mathrm{H}-14\right), 5.13$ (ddt, $1 \mathrm{H},{ }^{3} J=10.8,{ }^{3} J=3.3,{ }^{4} J=2.0, \mathrm{H}-$ 10), 5.21 (ddt, $1 \mathrm{H},{ }^{3} J=10.8,{ }^{3} J=2.2,{ }^{4} J=2.2, \mathrm{H}-9$ ), 9.01 (br s, $1 \mathrm{H}, \mathrm{H}$-pyrrole); ${ }^{13} \mathrm{C}$ NMR (assignment by HSQC, HMBC, $\left.600 \mathrm{MHz}, \mathrm{CDCl}_{3}\right): \delta=9.1\left(+, \mathrm{CH}_{3}\right), 14.5(-, \mathrm{C}-16), 22.6$ $\left(+,\left(\mathrm{CH}_{3}\right)_{3}\right), 24.9(-, \mathrm{C}-11), 25.5(-, \mathrm{C}-12), 26.5(-, \mathrm{C}-8)$, 31.1 (-, C-7), $35.2(-, \mathrm{C}-13), 51.8(+, \mathrm{C}-14), 55.6\left(\mathrm{C}_{\text {quat }}\right.$, $\left.\mathrm{C}\left(\mathrm{CH}_{3}\right)_{3}\right), 59.9$ (-, C-15), 70.9 (-, C-6), $111.8\left(\mathrm{C}_{\text {quat }}, \mathrm{C}-2\right)$, $120.0\left(\mathrm{C}_{\text {quat }}, \mathrm{C}-4\right), 128.5$ (+, C-10), 130.2 (+, C-9), 131.0 $\left(\mathrm{C}_{\text {quat }}, \mathrm{C}-5\right), 150.0\left(\mathrm{C}_{\text {quat }}, \mathrm{C}-3\right), 160.6\left(\mathrm{C}_{\text {quat }}, \mathrm{C}\right.$-ester $)$; $\mathrm{MS}$ (ES, $\left.\quad \mathrm{DCM} / \mathrm{MeOH}+10 \mathrm{mmol} / \mathrm{L} \quad \mathrm{NH}_{4} \mathrm{Ac}\right): \quad \mathrm{m} / \mathrm{z} \quad(\%)=411.2$ (100) $[\mathrm{M}+\mathrm{H}]^{+}, 821.6(13)[2 \mathrm{M}+\mathrm{H}]^{+}$; HRMS calcd for $\mathrm{C}_{21} \mathrm{H}_{34} \mathrm{~N}_{2} \mathrm{O}_{4} \mathrm{~S}\left[\mathrm{M}^{+\cdot}\right.$ ]: 410.2232; found: 410.2233 $\pm 1.5 \mathrm{ppm}$.

\subsubsection{Compound 29}

MS (ES, DCM/MeOH+10 mmol/L $\left.\mathrm{NH}_{4} \mathrm{Ac}\right): \mathrm{m} / \mathrm{z}(\%)=$ 819.7 (100) $\left[\mathrm{M}-\mathrm{H}^{+}\right]^{-}, 933.6$ (10) $[\mathrm{M}+\mathrm{TFA}]^{-}, 879.7$ (9) $\left[\mathrm{M}+\mathrm{CH}_{3} \mathrm{COO}^{-}\right], 855.6(8)\left[\mathrm{M}+\mathrm{Cl}^{-}\right]$.

\section{Acknowledgements}

We thank the Fonds der Chemischen Industrie and the Universität Regensburg for support. G.D. thanks the European Commission for a research travel grant (COST program).

\section{References and notes}

1. Eliel, E.; Wilen, S. H. Stereochemistry of Organic Compounds; WileyInterscience: New York, NY, 1994.

2. Lüttringhaus, A.; Gralheer, H. Liebigs Ann. 1947, 557, 112-120; Lüttringhaus, A.; Gralheer, H. Ann. 1947, 557, 108-112.

3. Lüttringhaus, A.; Gralheer, H. Liebigs Ann. 1941, 550, 67-98.

4. For an example, see: Paley, R. S. Chem. Rev. 2002, 102, 1493-1523; Paley, R. S.; Estroff, L. A.; Gauguet, J.-M.; Hunt, D. K.; Newlin, R. C. Org. Lett. 2000, 2, 365-368; Comely, A. C.; Gibson, S. E. J. Chem. Soc., Perkin Trans. 1 1999, 223-238.

5. For an example, see: Liu, D.; Xie, F.; Zhang, W. Tetrahedron Lett. 2007, 48, 585-588; Djukic, J.-P.; Michon, C.; Maisse-Francois, A.; Allagapen, R.; Pfeffer, M.; Dotz, K. H.; De Cian, A.; Fischer, J. Chem.-Eur. J. 2000, 6, 1064-1077.

6. Tanaka, K.; Sagae, H.; Toyoda, K.; Noguchi, K.; Hirano, M. J. Am. Chem. Soc. 2007, 129, 1522-1523; Aly, A. A.; Hopf, H.; Jones, P. G.; Dix, I. 
Tetrahedron 2006, 62, 4498-4505; Rajakumar, P.; Swaroop, M. G. Tetrahedron Lett. 2006, 47, 3019-3022; Ueda, T.; Kanomata, N.; Machida, H. Org. Lett. 2005, 7, 2365-2368.

7. For an example, see: Fürstner, A.; Szillat, H.; Gabor, B.; Mynott, R. J. Am. Chem. Soc. 1998, 120, 8305-8314.

8. Marchand, A.; Maxwell, A.; Mootoo, B.; Pelter, A.; Reid, A. Tetrahedron 2000, 56, 7331-7338; Pelter, A.; Kidwell, H.; Crump, R. A. N. C. J. Chem. Soc., Perkin Trans. 1 1997, 3137-3140; Pelter, A.; Crump, R. A. N. C.; Kidwell, H. Tetrahedron Lett. 1996, 37, 1273-1276.

9. Pelter, A.; Crump, R. A. N. C.; Kidwell, H. Tetrahedron: Asymmetry 1997, 8, 3873-3880.

10. Handbook of Metathesis; Grubbs, R. H., Ed.; Wiley-VCH: Weinheim, 2003; Alkene Metathesis in Organic Synthesis; Fuerstner, A., Ed.; Springer: Berlin, 1998; Connon, S. J.; Blechert, S. Angew. Chem., Int. Ed. 2003, 42, 1900-1923; Grubbs, R. H.; Miller, S. J.; Fu, G. C. Acc. Chem. Res. 1995, 28, 446-452; Furstner, A. Angew. Chem., Int. Ed. 2000, 39, 3012-3043.

11. For examples, see: El-azizi, Y.; Schmitzer, A.; Collins, S. K. Angew. Chem., Int. Ed. 2006, 45, 968-973; Watson, M. D.; Jaeckel, F.; Severin, N.; Rabe, J. P.; Muellen, K. J. Am. Chem. Soc. 2004, 126, 1402-1407; Martinez, V.; Blais, J.-C.; Bravic, G.; Astruc, D. Organometallics 2004, 23, 861-874; Fuerstner, A.; Stelzer, F.; Rumbo, A.; Krause, H. Chem.-Eur. J. 2002, 8, 1856-1871; Smith, A. B., III; Adams, C. M.; Kozmin, S. A. J. Am. Chem. Soc. 2001, 123, 990-991.

12. Fürstner, A.; Gastner, T.; Weintritt, H. J. Org. Chem. 1999, 64, 2361-2366.

13. The high temperature required for racemization does not permit determining the energy of the barrier experimentally, e.g., by variable temperature NMR measurements.

14. Hochmuth, D. H.; Koenig, W. A. Liebigs Ann. 1996, 947-951.

15. Bonauer, C.; Walenzyk, T.; Koenig, B. Synthesis 2006, 1-20; Bonauer, C.; Koenig, B. Synthesis 2005, 2367-2372.

16. Bonauer, C.; Zabel, M.; Koenig, B. Org. Lett. 2004, 6, 1349-1352.

17. Maity, P.; Koenig, B. Synthesis 2006, 2719-2724.

18. Chong, R.; Clezy, P. S. Aust. J. Chem. 1967, 20, 935-950.

19. Liu, G.; Cogan, D. A.; Owens, T. D.; Tang, T. P.; Ellman, J. A. J. Org. Chem. 1999, 64, 1278-1284.

20. Jiang, Z.-Y.; Chan, W. H.; Lee, A. W. M.J. Org. Chem. 2005, 70, 1081-1083.

21. McMahon, J. P.; Ellman, J. A. Org. Lett. 2004, 6, 1645-1647; Mukade, T.; Dragoli, D. R.; Ellman, J. A. J. Comb. Chem. 2003, 5, 590-596; Evans, J. W.; Ellman, J. A. J. Org. Chem. 2003, 68, 9948-9957; Plobeck, N.; Powell, D. Tetrahedron: Asymmetry 2002, 13, 303-310; Tang, T. P.; Volkman, S. K.; Ellman, J. A. J. Org. Chem. 2001, 66, 8772-8778; Bailey, P. D.; Clayson, J.; Boa, A. N. Contemp. Org. Synth. 1995, 2, 173-187.

22. Armstrong, S. K. J. Chem. Soc., Perkin Trans. 1 1998, 371-388; Grubbs, R. H.; Chang, S. Tetrahedron 1998, 54, 4413-4450.

23. The $N$-Boc-protected derivative 23-Boc was tested to explore the effect of the pyrrole amine function on the RCM reaction. However, reactions with and without $N$-Boc protection of the pyrrole nitrogen atom gave equal results.
24. This catalyst was tested to prevent the possible formation of a chelate intermediate between the tert-butyldimethylsilyl (TBS) protecting group and the ruthenium ion trapping the catalyst in an inactive form. This chelation should be avoided by the chelating effect of the isopropoxy-group of the second generation Hoveyda-Grubb's catalyst: Zaja, M.; Connon, S. J.; Dunne, A. M.; Rivard, M.; Buschmann, N.; Jiricek, J.; Blechert, S. Tetrahedron 2003, 59, 6545-6558.

25. Cross metathesis processes generally lead to the thermodynamically most stable members of a set of structurally related isomers: Smith, A. B., III; Adams, C. M.; Kozmin, S. A.; Paone, D. V. J. Am. Chem. Soc. 2001, 123, $5925-5937$

26. The prediction of alkene geometry in ring-closing ene-ene metathesis is still difficult: Arisawa, M.; Nishida, A.; Nakagawa, M. J. Organomet Chem. 2006, 691, 5109-5121; Fürstner, A.; Dierkes, T.; Thiel, O. R.; Blanda, G. Chem.-Eur. J. 2001, 7, 5286-5298; Fürstner, A.; Thiel, O. R.; Ackermann, L. Org. Lett. 2001, 3, 449-451; Lee, C. W.; Grubbs, R. H. Org. Lett. 2000, 2, 2145-2147; Fürstner, A.; Thiel, O. R.; Kindler, N.; Bartkowska, B. J. Org. Chem. 2000, 65, 7990-7995; Fürstner, A.; Thiel, O. R.; Blanda, G. Org. Lett. 2000, 2, 3731-3734.

27. (a) Lam, P. C.-H.; Carlier, P. R. J. Org. Chem. 2005, 70, 1530-1538; (b) Mitchell, R. H.; Blunden, R.; Hollett, G.; Bandyopadhyay, S.; Vaughan Williams, R.; Twamley, B. J. Org. Chem. 2005, 70, 675-680.

28. HPLC analysis of the RCM product from diene 23-Boc on a chiral column showed four isomeric compounds (one major, three minor). This again indicates a stereochemical bias of the ring-closing reaction.

29. Frisch, M. J.; Trucks, G. W.; Schlegel, H. B.; Scuseria, G. E.; Robb, M. A.; Cheeseman, J. R.; Montgomery, J. A., Jr.; Vreven, T.; Kudin, K. N.; Burant, J. C.; Millam, J. M.; Iyengar, S. S.; Tomasi, J.; Barone, V.; Mennucci, B.; Cossi, M.; Scalmani, G.; Rega, N.; Petersson, G. A.; Nakatsuji, H.; Hada, M.; Ehara, M.; Toyota, K.; Fukuda, R.; Hasegawa, J.; Ishida, M.; Nakajima, T.; Honda, Y.; Kitao, O.; Nakai, H.; Klene, M.; Li, X.; Knox, J. E.; Hratchian, H. P.; Cross, J. B.; Bakken, V.; Adamo, C.; Jaramillo, J.; Gomperts, R.; Stratmann, R. E.; Yazyev, O.; Austin, A. J.; Cammi, R.; Pomelli, C.; Ochterski, J. W.; Ayala, P. Y.; Morokuma, K.; Voth, G. A.; Salvador, P.; Dannenberg, J. J.; Zakrzewski, V. G.; Dapprich, S.; Daniels, A. D.; Strain, M. C.; Farkas, O.; Malick, D. K.; Rabuck, A. D.; Raghavachari, K.; Foresman, J. B.; Ortiz, J. V.; Cui, Q.; Baboul, A. G.; Clifford, S.; Cioslowski, J.; Stefanov, B. B.; Liu, G.; Liashenko, A.; Piskorz, P.; Komaromi, I.; Martin, R. L.; Fox, D. J.; Keith, T.; Al-Laham, M. A.; Peng, C. Y.; Nanayakkara, A.; Challacombe, M.; Gill, P. M. W.; Johnson, B.; Chen, W.; Wong, M. W.; Gonzalez, C.; Pople, J. A. Gaussian 03, Revision D.02; Gaussian: Wallingford, CT, 2004.

30. (a) Becke, A. D. Phys. Rev. A 1988, 38, 3098-3100; (b) Becke, A. D J. Chem. Phys. 1993, 98, 5648-5652. The Lee-Yang-Parr nonlocal correlation functional: (c) Lee, C.; Yang, W.; Parr, R. G. Phys. Rev. B 1988, 37, 785-789.

31. Hehre, W. J.; Ditchfield, R.; Pople, J. A. J. Chem. Phys. 1972, 56, 22572261. 